\title{
A Reevaluation of Prazosin Pharmacokinetics in a Two-Compartment Model, the Apparent Volume of Distribution and Comparative Simulations in the One-Compartment Model
}

\author{
Michalakis Savva ${ }^{1}$, Xudong Yuan ${ }^{2}$ \\ ${ }^{1}$ South University, Savannah, USA \\ ${ }^{2}$ Acon Pharmaceuticals, Cranbury, USA \\ Email: ^msavva@southuniversity.edu, ^micsavva@gmail.com
}

How to cite this paper: Savva, M. and Yuan, X.D. (2022) A Reevaluation of Prazosin Pharmacokinetics in a Two-Compartment Model, the Apparent Volume of Distribution and Comparative Simulations in the One-Compartment Model. Journal of Biosciences and Medicines, 10, 108-140. https://doi.org/10.4236/jbm.2022.101010

Received: December 17, 2021

Accepted: January 23, 2022

Published: January 26, 2022

Copyright $\odot 2022$ by author(s) and Scientific Research Publishing Inc. This work is licensed under the Creative Commons Attribution International License (CC BY 4.0).

http://creativecommons.org/licenses/by/4.0/

(c) (i) Open Access

\begin{abstract}
Published clinical data of Prazosin were reevaluated pharmacokinetically using explicit solutions to drug concentration as a function of total time for IV bolus injection, intermittent intravenous infusion and oral routes of administration in an open two-compartment model. In a novel way, the apparent volume of distribution was estimated from a two-compartment model and found to be close to the total body water suggesting that Prazosin is distributed in all tissues both extracellularly and intracellularly. In addition, extracting the value of the apparent volume of distribution from a two-compartment model allowed comparative simulations in the one-compartment model. It is shown that dosage calculations of Prazosin intermittent infusion can be safely performed using the simpler one-compartment model equations. Lastly, several additional time-dependent pharmacokinetic parameters e.g., the peak time in the central and peripheral compartment and nonsteady state and steady state peak concentration and AUC were determined using series equations for all three routes of administration, as a function of dose number and total time upon multiple drug administrations in the two-compartment model. It is also the first time that steady-state plasma drug concentration equations were derived in a two-compartment mammillary model.
\end{abstract}

\section{Keywords}

Prazosin, Pharmacokinetics, Intravenous Bolus, Intermittent Infusion, Oral Dose, Multiple Doses, Compartment Model, Apparent Volume of Distribution 


\section{Introduction}

Despite their primary role in estimating pharmacokinetic parameters that can be used to design optimum dosing regimens to achieve plasma drug concentrations within therapeutic range, it is generally believed that the apparent volume of distribution cannot be estimated in multi-compartment models and that the compartment volumes of multi-compartment pharmacokinetic models bear no physiological significance. This is no longer true [1] [2] [3]. The volume of distribution of a substance in a phase is the volume of that phase in the system. The apparent volume of distribution $\left(V_{d}\right)$ of a solute associated with a solvent in a two-phase system on the other hand, can be determined at equilibrium using a mass balance equation (Equation (1)).

$$
V_{d, 1}=V_{1}+K_{2,1} \cdot V_{2}
$$

In pharmacokinetic modeling, each compartment has its own solute's $V_{d}$ only when the compartments constitute different phases. Chemically or compositionally similar, kinetically different, compartments have the same $V_{d}$ which is equal to the total volume of the kinetically different compartments. As with the partition coefficient, there are as many solute $V_{d}$ as the number of phases in a system. Unlike the partition coefficient, however, the value of the $V_{d}$ of a substance is dependent on the actual phase volumes, and at constant phase volumes, phase solute concentrations are directly proportional to the total mass of the solute $x_{s}$ in the system as shown by Equation (2), below:

$$
\begin{aligned}
& V_{d, 1}=\frac{x_{s}}{x_{1}} \cdot V_{1} \\
& V_{d, 2}=\frac{x_{s}}{x_{2}} \cdot V_{2}
\end{aligned}
$$

In vivo, the apparent volume of distribution of a drug can be defined as the volume of the assayed phase that is needed to dissolve the whole drug dose and achieve the measured drug concentration in the assayed phase. Its value depends on the physicochemical properties of the drug and the body's physiology which regulates the chemical constituency and the actual fluid volumes of the pharmacokinetic compartments. The presence of disease states and other pathological conditions may cause deviations from what we usually measure in normal healthy individuals. Under constant conditions, the apparent volume of distribution is a constant parameter and thus, in accordance with Equation (2) increasing or decreasing a dose $\left(x_{s}\right)$ will result in an increase or decrease of plasma drug concentration, respectively. Drug concentrations are usually measured in plasma but the $V_{d}$ of a drug is related to the whole compartment that contains the plasma along with other body fluids. Since changes in drug dose are reflected in changes of drug concentration in the assayed compartment, Equation (2) can be used to compute the $V_{d}$ from the administered dose and the measured drug concentration in the assayed compartment. We have carried out such an analysis on clinical data of the drug sisomicin in an open two-compartment pharmacoki- 
netic model and have determined the apparent volume of distribution of the drug. In addition, we were able to show that the volumes of the central and peripheral compartment are approximately equal to the extracellular fluid volume and thus, they represent the true distribution of sisomicin in the body [3].

Prazosin was first used in the mid-seventies as an antihypertensive agent due to its alpha-1 receptor blocking properties in vascular smooth muscle of arteries and veins [4]. A number of clinical studies assessed some of the pharmacokinetic properties of the drug [5]-[13]. Most of the single-dose studies support a twocompartment open model for prazosin's in vivo disposition. One of the early clinical studies of Prazosin was that of Bateman and coworkers. These investigators have determined pharmacokinetic micro rate constants and the hybrid distribution and elimination rate constants in a two-compartment model but they were unable to discern the physiological relevance of the central and peripheral compartment volumes in their model. Although the drug was administered by a 10-minute intermittent infusion, results were analyzed using the explicit solution to drug concentration after IV bolus administration and only for the central compartment using the so-called mid-point of the infusion rule. The only pharmacokinetic parameters extracted from clinical studies conducted with an oral dosage form (Hypovase) were the bioavailability factor and the half-life of drug elimination [5]. The investigators were neither able to assess parameters of the peripheral compartment nor time-dependent pharmacokinetic parameters, such as, the peak time and peak concentration in the central and peripheral compartments. Similarly, a clinical study conducted with a single intravenous bolus dose of prazosin concluded that the drug follows a two-compartment kinetic model [6]. Several useful pharmacokinetic parameters were determined but not the $V_{d}$, the $V_{2}$, the drug concentration, AUC, peak time and peak concentration in the peripheral compartment.

We have recently derived series equations for various routes of drug administration that simulate drug concentration not just within the first dosing interval, but in the whole time-domain of therapy [3] [14] [15]. We have additionally produced herein, the real-time explicit solutions to drug concentration for multiple IV boluses and multiple oral doses in a two-compartment model and utilized them along with the analytical solutions of the multiple intermittent infusions that we have reported elsewhere to analyze published clinical data of prazosin. The goal was to assess various primary and secondary time-dependent pharmacokinetic parameters, as well, as to simulate non-steady state and steady state concentration-time curves in both central and peripheral compartments upon administration of multiple doses. In particular, we have achieved the construction of the accumulation phase of the intermittent intravenous infusion of Prazosin from pharmacokinetic parameters extracted from the elimination phase of the drug. In addition, the assessment of Prazosin's apparent volume of distribution from a two-compartment model enabled comparative simulations of the one-compartment open model for all three routes of drug administration. 


\section{Methods}

\subsection{Explanation of Terms}

$V_{d}$ : Drug apparent volume of distribution

CL: Drug clearance

$k$. Drug first-order elimination rate constant

$\tau$ : dosing interval

$D$. Drug dose

$n$ : number of doses

$x_{1,1}$ : Drug amount in central or compartment 1 during the first dose $(0 \leq t \leq \tau)$.

$x_{1,2}$ : Drug amount in compartment 1 during the second dose $(\tau \leq t \leq 2 \cdot \tau)$.

$x_{2,1}$ : Drug amount in the peripheral or compartment 2 during the first dose $(0 \leq t \leq \tau)$.

$C_{1,1, \max }$ : Maximum drug concentration in compartment 1 after administration of the first dose.

$C_{2,3 \text { max }}$ : Maximum drug concentration in compartment 2 after administration of the third dose.

$C_{1,3, \min }:$ Minimum drug concentration in compartment 1 after the third dose at $t=3 \cdot \tau$.

$C_{2,4, \min }$ : Minimum drug concentration in the peripheral compartment at the end of the fourth dosing interval $(t=4 \cdot \tau)$.

$C_{2, n, s s, t}$ : Steady state drug concentration in the peripheral compartment as a function of dose number and total time.

\subsection{Compartment Models}

Explicit solutions to concentration were developed for multiple IV boluses and multiple oral administration in an open two-compartment mammillary model (Figure 1) following an approach that was recently described elsewhere [3] [14] [15].

\subsubsection{Multiple Intravenous Boluses in an Open Two-Compartment Mammillary Model \\ Differential equations for inputs and outputs.}

Input: The whole drug dose $(D)$ is transferred into the central compartment instantly.

Output: First-order drug elimination from the central compartment.

$$
\begin{gathered}
\dot{x}_{1}=-\left(k_{10}+k_{12}\right) \cdot x_{1}+k_{21} \cdot x_{2} ; \quad x_{1}(0)=D \\
\dot{x}_{2}=k_{12} \cdot x_{1}-k_{21} \cdot x_{2} ; \quad x_{2}(0)=0
\end{gathered}
$$

Analytical solutions to drug amount in the central and peripheral compartments after a single intravenous bolus injection

Using Laplace transform to the initial value problem and writing $X_{1}$ and $X_{2}$ for $\mathcal{L}\left\{x_{1}(t)\right\}$ and $\mathcal{L}\left\{x_{2}(t)\right\}$, we obtain: 


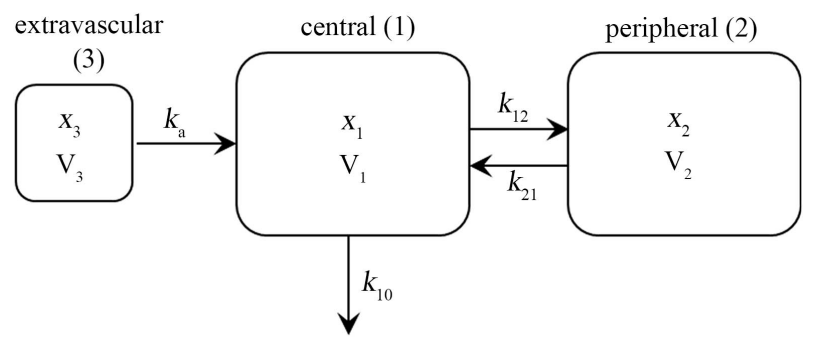

Figure 1. Extravascular drug administration in a two-compartment mammillary model. Drug absorption and elimination is described by first-order kinetics into and out of the central compartment, respectively.

$$
\begin{aligned}
& \left(s+k_{10}+k_{12}\right) \cdot X_{1}-k_{21} \cdot X_{2}=D \\
& -k_{12} \cdot X_{1}+\left(s+k_{21}\right) \cdot X_{2}=0
\end{aligned}
$$

Solving Equation (6) for $X_{1}$ and substituting on Equation (5),

$$
X_{2}=\frac{D \cdot k_{12}}{\left(s^{2}+\left(k_{10}+k_{12}+k_{21}\right) \cdot s+k_{10} \cdot k_{12}\right)}
$$

Let $\lambda_{1}+\lambda_{2}=k_{10}+k_{12}+k_{21}$ and $\lambda_{1} \cdot \lambda_{2}=k_{10} \cdot k_{21}$

$$
X_{2}=\frac{D \cdot k_{12}}{\left(s+\lambda_{1}\right) \cdot\left(s+\lambda_{2}\right)}=\frac{A}{\left(s+\lambda_{1}\right)}+\frac{B}{\left(s+\lambda_{2}\right)}
$$

Using the method of partial fraction decomposition,

$$
\begin{gathered}
A=\frac{D \cdot k_{12}}{\left(\lambda_{2}-\lambda_{1}\right)} ; B=-\frac{D \cdot k_{12}}{\left(\lambda_{2}-\lambda_{1}\right)} \\
X_{2}=\frac{D \cdot k_{12}}{\left(\lambda_{2}-\lambda_{1}\right)} \cdot\left(\frac{1}{s+\lambda_{1}}-\frac{1}{s+\lambda_{2}}\right)
\end{gathered}
$$

Taking the inverse transform,

$$
\begin{gathered}
\mathcal{L}^{-1}\left\{X_{2}(s)\right\}=x_{2}(t)=D \cdot k_{12} \cdot\left(A_{2} \cdot \mathrm{e}^{-\lambda_{1} \cdot t}+B_{2} \cdot \mathrm{e}^{-\lambda_{2} \cdot t}\right) \\
A_{2}=\frac{1}{\left(\lambda_{2}-\lambda_{1}\right)} ; \quad B_{2}=\frac{1}{\left(\lambda_{1}-\lambda_{2}\right)}
\end{gathered}
$$

Substituting $x_{2}(t)$ on Equation (4),

$$
\begin{gathered}
x_{1}(t)=D \cdot\left(A_{1} \cdot \mathrm{e}^{-\lambda_{1} \cdot t}+B_{1} \cdot \mathrm{e}^{-\lambda_{2} \cdot t}\right) \\
A_{1}=\frac{\lambda_{1}-k_{21}}{\left(\lambda_{1}-\lambda_{2}\right)} ; \quad B_{1}=\frac{k_{21}-\lambda_{2}}{\left(\lambda_{1}-\lambda_{2}\right)} ; \\
C_{1}(t)=\frac{x_{1}(t)}{V_{1}}
\end{gathered}
$$

Terms of the mathematical sequence for multiple IV doses administered with a dosing interval $\tau$.

$$
\begin{aligned}
\left\{C_{1, n}\right\}= & \left\{\frac{D}{V_{1}} \cdot\left(A_{1} \cdot \mathrm{e}^{-\lambda_{1} \cdot t}+B_{1} \cdot \mathrm{e}^{-\lambda_{2} \cdot t}\right), \frac{D}{V_{1}} \cdot\left(A_{1} \cdot \mathrm{e}^{-\lambda_{1} \cdot t}+B_{1} \cdot \mathrm{e}^{-\lambda_{2} \cdot t}\right)\right. \\
& \left.+\frac{D}{V_{1}} \cdot\left(A_{1} \cdot \mathrm{e}^{-\lambda_{1} \cdot(t-\tau)}+B_{1} \cdot \mathrm{e}^{-\lambda_{2} \cdot(t-\tau)}\right), \cdots\right\}
\end{aligned}
$$




\section{Pattern of Sequence}

$$
\left\{C_{1, n}\right\}=\left\{\frac{D}{V_{1}} \cdot\left(A_{1} \cdot \mathrm{e}^{-\lambda_{1} \cdot(t-(n-1) \cdot \tau)}+B_{1} \cdot \mathrm{e}^{-\lambda_{2} \cdot(t-(n-1) \cdot \tau)}\right)\right\}
$$

where, $t$ is the total time.

Partial sums and formula of the mathematical Series for repetitive IV bolus doses

$$
C_{1, n, t}=\sum_{n=1}^{\infty} \frac{D}{V_{1}} \cdot\left(A_{1} \cdot \mathrm{e}^{-\lambda_{1} \cdot(t-(n-1) \cdot \tau)}+B_{1} \cdot \mathrm{e}^{-\lambda_{2} \cdot(t-(n-1) \cdot \tau)}\right)
$$

Multiplying and dividing the two terms of the above equation with $\left(1-\mathrm{e}^{\lambda_{1} \cdot \tau}\right)$ and $\left(1-\mathrm{e}^{\lambda_{2} \cdot \tau}\right)$, respectively,

$$
\begin{gathered}
C_{1, n, t}=\frac{D}{V_{1}} \cdot\left\{A_{1} \cdot \frac{\left(1-\mathrm{e}^{-\lambda_{1} \cdot n \cdot \tau}\right)}{\left(1-\mathrm{e}^{-\lambda_{1} \cdot \tau}\right)} \cdot \mathrm{e}^{-\lambda_{1} \cdot(t-(n-1) \cdot \tau)}+B_{1} \cdot \frac{\left(1-\mathrm{e}^{-\lambda_{2} \cdot n \cdot \tau}\right)}{\left(1-\mathrm{e}^{-\lambda_{2} \cdot \tau}\right)} \cdot \mathrm{e}^{-\lambda_{2} \cdot(t-(n-1) \cdot \tau)}\right\} \\
C_{2, n, t}=\frac{D \cdot k_{12}}{V_{2}} \cdot\left\{A_{2} \cdot \frac{\left(1-\mathrm{e}^{-\lambda_{1} \cdot n \cdot \tau}\right)}{\left(1-\mathrm{e}^{-\lambda_{1} \cdot \tau}\right)} \cdot \mathrm{e}^{-\lambda_{1} \cdot(t-(n-1) \cdot \tau)}+B_{2} \cdot \frac{\left(1-\mathrm{e}^{-\lambda_{2} \cdot n \cdot \tau}\right)}{\left(1-\mathrm{e}^{-\lambda_{2} \cdot \tau}\right)} \cdot \mathrm{e}^{-\lambda_{2} \cdot(t-(n-1) \cdot \tau)}\right\}
\end{gathered}
$$

The corresponding steady-state concentrations of the drug in the two compartments are,

$$
\begin{gathered}
C_{1, n, s s, t}=\frac{D}{V_{1}} \cdot\left\{\frac{A_{1}}{\left(1-\mathrm{e}^{-\lambda_{1} \cdot \tau}\right)} \cdot \mathrm{e}^{-\lambda_{1} \cdot(t-(n-1) \cdot \tau)}+\frac{B_{1}}{\left(1-\mathrm{e}^{-\lambda_{2} \cdot \tau}\right)} \cdot \mathrm{e}^{-\lambda_{2} \cdot(t-(n-1) \cdot \tau)}\right\} \\
C_{2, n, s s, t}=\frac{D \cdot k_{12}}{V_{2}} \cdot\left\{\frac{A_{2}}{\left(1-\mathrm{e}^{-\lambda_{1} \cdot \tau}\right)} \cdot \mathrm{e}^{-\lambda_{1} \cdot(t-(n-1) \cdot \tau)}+\frac{B_{2}}{\left(1-\mathrm{e}^{-\lambda_{2} \cdot \tau}\right)} \cdot \mathrm{e}^{-\lambda_{2} \cdot(t-(n-1) \cdot \tau)}\right\}
\end{gathered}
$$

Notice that the drug concentrations at time zero after administration of the first dose $(n=1)$ in the central and peripheral compartments are,

$$
\begin{gathered}
C_{1,1,0}=C_{1,1, \max }=\frac{D}{V_{1}} \\
C_{2,1,0}=0
\end{gathered}
$$

Trough concentrations can be obtained from Equations (9)-(12) at $t=n \cdot \tau$.

$$
\begin{gathered}
C_{1, n, \min }=\frac{D}{V_{1}} \cdot\left\{A_{1} \cdot \frac{\left(1-\mathrm{e}^{-\lambda_{1} \cdot n \cdot \tau}\right)}{\left(1-\mathrm{e}^{-\lambda_{1} \cdot \tau}\right)} \cdot \mathrm{e}^{-\lambda_{1} \cdot \tau}+B_{1} \cdot \frac{\left(1-\mathrm{e}^{-\lambda_{2} \cdot n \cdot \tau}\right)}{\left(1-\mathrm{e}^{-\lambda_{2} \cdot \tau}\right)} \cdot \mathrm{e}^{-\lambda_{2} \cdot \tau}\right\} n \in[0, \infty) \\
C_{2, n, \min }=\frac{D \cdot k_{12}}{V_{2}} \cdot\left\{A_{2} \cdot \frac{\left(1-\mathrm{e}^{-\lambda_{1} \cdot n \cdot \tau}\right)}{\left(1-\mathrm{e}^{-\lambda_{1} \cdot \tau}\right)} \cdot \mathrm{e}^{-\lambda_{1} \cdot \tau}+B_{2} \cdot \frac{\left(\left(1-\mathrm{e}^{-\lambda_{2} \cdot n \cdot \tau}\right)\right)}{\left(1-\mathrm{e}^{-\lambda_{2} \cdot \tau}\right)} \cdot \mathrm{e}^{-\lambda_{2} \cdot \tau}\right\} n \in[0, \infty) \\
C_{1, \min , s s}=\frac{D}{V_{1}} \cdot\left\{\frac{A_{1}}{\left(1-\mathrm{e}^{-\lambda_{1} \cdot \tau}\right)} \cdot \mathrm{e}^{-\lambda_{1} \cdot \tau}+\frac{B_{1}}{\left(1-\mathrm{e}^{-\lambda_{2} \cdot \tau}\right)} \cdot \mathrm{e}^{-\lambda_{2} \cdot \tau}\right\} \\
C_{2, \min , s s}=\frac{D \cdot k_{12}}{V_{2}} \cdot\left\{\frac{A_{2}}{\left(1-\mathrm{e}^{-\lambda_{1} \cdot \tau}\right)} \cdot \mathrm{e}^{-\lambda_{1} \cdot \tau}+\frac{B_{2}}{\left(1-\mathrm{e}^{-\lambda_{2} \cdot \tau}\right)} \cdot \mathrm{e}^{-\lambda_{2} \cdot \tau}\right\}
\end{gathered}
$$




\section{AUC formulas}

The areas under the curve per dosing interval were obtained by integration of the drug concentration from $(n-1) \cdot \tau$ to $n \cdot \tau$.

$$
\begin{aligned}
\mathrm{AUC}_{1, n, \tau}= & \frac{D}{V_{1}} \cdot\left[A_{1} \cdot \frac{\left(1-\mathrm{e}^{-\lambda_{1} \cdot n \cdot \tau}\right)}{\lambda_{1}}+B_{1} \cdot \frac{\left(1-\mathrm{e}^{-\lambda_{2} \cdot n \cdot \tau}\right)}{\lambda_{2}}\right] \\
\mathrm{AUC}_{2, n, \tau}= & \frac{D \cdot k_{12}}{V_{2}} \cdot\left[A_{2} \cdot \frac{\left(1-\mathrm{e}^{-\lambda_{1} \cdot n \cdot \tau}\right)}{\lambda_{1}}+B_{2} \cdot \frac{\left(1-\mathrm{e}^{-\lambda_{2} \cdot n \cdot \tau}\right)}{\lambda_{2}}\right] \\
& \mathrm{AUC}_{1, \tau, s s}=\frac{D}{V_{1}} \cdot\left[\frac{A_{1}}{\lambda_{1}}+\frac{B_{1}}{\lambda_{2}}\right] \\
\mathrm{AUC}_{2, \tau, s s}= & \frac{D \cdot k_{12}}{V_{2}} \cdot\left[\frac{A_{2}}{\lambda_{1}}+\frac{B_{2}}{\lambda_{2}}\right]
\end{aligned}
$$

The maximum drug concentration in the central compartment can be determined from Equation (9) as a function of bolus dose at $t=(n-1) \cdot \tau$.

$$
\begin{gathered}
C_{1, n, \max }=\frac{D}{V_{1}} \cdot\left\{A_{1} \cdot \frac{\left(1-\mathrm{e}^{-\lambda_{1} \cdot n \cdot \tau}\right)}{\left(1-\mathrm{e}^{-\lambda_{1} \cdot \tau}\right)}+B_{1} \cdot \frac{\left(1-\mathrm{e}^{-\lambda_{2} \cdot n \cdot \tau}\right)}{\left(1-\mathrm{e}^{-\lambda_{2} \cdot \tau}\right)}\right\} \\
C_{1, s s, \text { max }}=\frac{D}{V_{1}} \cdot\left[\frac{A_{1}}{\left(1-\mathrm{e}^{-\lambda_{1} \cdot \tau}\right)}+\frac{B_{1}}{\left(1-\mathrm{e}^{-\lambda_{2} \cdot \tau}\right)}\right]
\end{gathered}
$$

In order to obtain the maximum drug concentration in the peripheral compartment upon multiple IV boluses we first need to determine the peripheral compartment peak time as a function of intravenous bolus dose $\left(t_{2, n \text {, max }}\right)$ from Equation (4) (Appendix).

$$
\begin{gathered}
t_{2, n, \max }=(n-1) \cdot \tau+\frac{\ln \left(\frac{\lambda_{2} \cdot\left(1-\mathrm{e}^{-\lambda_{2} \cdot n \cdot \tau}\right) \cdot\left(1-\mathrm{e}^{-\lambda_{1} \cdot \tau}\right)}{\lambda_{1} \cdot\left(1-\mathrm{e}^{-\lambda_{2} \cdot \tau}\right) \cdot\left(1-\mathrm{e}^{-\lambda_{1} \cdot n \cdot \tau}\right)}\right)}{\left(\lambda_{2}-\lambda_{1}\right)} \\
C_{2, n, \max }=\frac{D \cdot k_{12}}{V_{2}} \cdot\left\{A_{2} \cdot \frac{\left(1-\mathrm{e}^{-\lambda_{1} \cdot n \cdot \tau}\right)}{\left(1-\mathrm{e}^{-\lambda_{1} \cdot \tau}\right)} \cdot \mathrm{e}^{-\lambda_{1} \cdot\left(t_{2, \max , n}-(n-1) \cdot \tau\right)}\right. \\
+B_{2} \cdot \frac{\left(1-\mathrm{e}^{-\lambda_{2} \cdot n \cdot \tau}\right)}{\left.\left(1-\mathrm{e}^{-\lambda_{2} \cdot \tau}\right) \cdot \mathrm{e}^{-\lambda_{2} \cdot\left(t_{2, \max , n}-(n-1) \cdot \tau\right)}\right\}} \\
t_{2, n, \text { max }, s s}=(n-1) \cdot \tau+\frac{\ln \left(\frac{\lambda_{2} \cdot\left(1-\mathrm{e}^{-\lambda_{1} \cdot \tau}\right)}{\lambda_{1} \cdot\left(1-\mathrm{e}^{-\lambda_{2} \cdot \tau}\right)}\right)}{\left(\lambda_{2}-\lambda_{1}\right)} \\
t_{2, \max , s s}^{*}=\frac{\ln \left(\frac{\lambda_{2} \cdot\left(1-\mathrm{e}^{-\lambda_{1} \cdot \tau}\right)}{\lambda_{1} \cdot\left(1-\mathrm{e}^{-\lambda_{2} \cdot \tau}\right)}\right)}{\left(\lambda_{2}-\lambda_{1}\right)}
\end{gathered}
$$

where $t_{2, \max , s s}^{*}$ is the steady state time within a dosing interval $0 \leq t_{2, \max , s s}^{*} \leq \tau$. 


$$
\begin{aligned}
C_{2, n, \max , s s}= & \frac{D \cdot k_{12}}{V_{2}} \cdot\left\{\frac{A_{2}}{\left(1-\mathrm{e}^{-\lambda_{1} \cdot \tau}\right)} \cdot \mathrm{e}^{-\lambda_{1} \cdot\left(t_{2, n, \max }-(n-1) \cdot \tau\right)}\right. \\
& \left.+\frac{B_{2}}{\left(1-\mathrm{e}^{-\lambda_{2} \cdot \tau}\right)} \cdot \mathrm{e}^{-\lambda_{2} \cdot\left(t_{2, n, \max }-(n-1) \cdot \tau\right)}\right\} \\
C_{2, \text { max }, s s}= & \frac{D \cdot k_{12}}{V_{2}} \cdot\left(\frac{A_{2}}{\left(1-\mathrm{e}^{-\lambda_{1} \cdot \tau}\right)} \cdot \mathrm{e}^{-\lambda_{1} \cdot t_{2, \max , s s}^{*}}+\frac{B_{2}}{\left(1-\mathrm{e}^{-\lambda_{2} \cdot \tau}\right)} \cdot \mathrm{e}^{-\lambda_{2} \cdot t_{2, \max , s s}^{*}}\right)
\end{aligned}
$$

At the peripheral compartment peak time there is an equal rate of exchange of drug molecules from the central to peripheral compartment and vice versa. Hence, the time of momentary distribution equilibrium, $t_{e q, n}$ where the apparent volume of distribution of the drug with respect to the central phase $\left(V_{d, 1}\right)$ can be determined, is the same as $t_{2, n, \max }$. Therefore,

$$
t_{2, n, \max }=t_{e q, n}
$$

The time of momentary distribution equilibrium during the first dosing interval $(n=1)$ is:

$$
t_{e q, 1}=\frac{\ln \left(\frac{\lambda_{2}}{\lambda_{1}}\right)}{\left(\lambda_{2}-\lambda_{1}\right)}
$$

The peak time of concentration in the peripheral compartment under steady-state conditions is,

$$
t_{e q, s s}=t_{2, \max , s s}^{*}=\frac{\ln \left(\frac{\lambda_{2}}{\lambda_{1}} \cdot \frac{\left(1-\mathrm{e}^{-\lambda_{1} \cdot \tau}\right)}{\left(1-\mathrm{e}^{-\lambda_{2} \cdot \tau}\right)}\right)}{\left(\lambda_{2}-\lambda_{1}\right)}
$$

The non-steady state drug concentration in the central compartment at momentary distribution equilibrium as a function of IV dose number is shown with Equation (29) whereas the steady state drug concentration in the central compartment at times of momentary distribution equilibrium is expressed by Equation (30).

$$
\begin{gathered}
C_{1, e q, n, t}=\frac{D}{V_{1}} \cdot\left\{A_{1} \cdot \frac{\left(1-\mathrm{e}^{-\lambda_{1} \cdot n \cdot \tau}\right)}{\left(1-\mathrm{e}^{-\lambda_{1} \cdot \tau}\right)} \cdot \mathrm{e}^{-\lambda_{1} \cdot\left(t_{2, \max , n}-(n-1) \cdot \tau\right)}\right. \\
\left.+B_{1} \cdot \frac{\left(1-\mathrm{e}^{-\lambda_{2} \cdot n \cdot \tau}\right)}{\left(1-\mathrm{e}^{-\lambda_{2} \cdot \tau}\right)} \cdot \mathrm{e}^{-\lambda_{2} \cdot\left(t_{2, \max , n}-(n-1) \cdot \tau\right)}\right\} \\
C_{1, e q, n, s s}=\frac{D}{V_{1}} \cdot\left\{\frac{A_{1}}{\left(1-\mathrm{e}^{-\lambda_{1} \cdot \tau}\right)} \cdot \mathrm{e}^{-\lambda_{1} \cdot t_{2, \max , s s}^{*}}+\frac{B_{1}}{\left(1-\mathrm{e}^{-\lambda_{2} \cdot \tau}\right)} \cdot \mathrm{e}^{-\lambda_{2} \cdot t_{2, \text { max }, s s}^{*}}\right\}
\end{gathered}
$$

\subsubsection{Multiple Oral Administration in a Two-Compartment Mammillary Model \\ Differential equations for inputs and outputs.}


Input: First-order drug absorption into the central compartment.

Output: First-order drug elimination from the central compartment.

$$
\begin{gathered}
\dot{x}_{1}=-\left(k_{10}+k_{12}\right) \cdot x_{1}+k_{21} \cdot x_{2}+k_{a} \cdot x_{3} ; \quad x_{1}(0)=0 \\
\dot{x}_{2}=k_{12} \cdot x_{1}-k_{21} \cdot x_{2} ; \quad x_{2}(0)=0 \\
\dot{x}_{3}=-k_{a} \cdot x_{3} ; \quad x_{3}(0)=D
\end{gathered}
$$

where $D$ is the drug dose and $k_{a}$ is the first-order absorption rate constant. Work related to the analytical solutions of Equations (31)-(33) is included in the Appendix. Only the final equations are shown in this section.

Analytical solutions to drug concentration after single oral doses

$$
\begin{aligned}
& x_{1}(t)=D \cdot k_{a} \cdot\left(A_{3} \cdot \mathrm{e}^{-\lambda_{1} \cdot t}+B_{3} \cdot \mathrm{e}^{-\lambda_{2} \cdot t}+E_{3} \cdot \mathrm{e}^{-k_{a} \cdot t}\right) \\
& A_{3}=\frac{\left(k_{21}-\lambda_{1}\right)}{\left(k_{a}-\lambda_{1}\right) \cdot\left(\lambda_{2}-\lambda_{1}\right)} ; \quad B_{3}=\frac{\left(k_{21}-\lambda_{2}\right)}{\left(k_{a}-\lambda_{2}\right) \cdot\left(\lambda_{1}-\lambda_{2}\right)} ; \quad E_{3}=\frac{\left(k_{21}-k_{a}\right)}{\left(k_{a}-\lambda_{1}\right) \cdot\left(k_{a}-\lambda_{2}\right)} \\
& C_{1}(t)=\frac{x_{1}(t)}{V_{1}} \\
& x_{2}(t)=D \cdot k_{a} \cdot k_{12} \cdot\left(A_{4} \cdot \mathrm{e}^{-\lambda_{1} \cdot t}+B_{4} \cdot \mathrm{e}^{-\lambda_{2} \cdot t}+E_{4} \cdot \mathrm{e}^{-k_{a} \cdot t}\right) \\
& A_{4}=\frac{1}{\left(k_{a}-\lambda_{1}\right) \cdot\left(\lambda_{2}-\lambda_{1}\right)} ; \quad B_{4}=\frac{1}{\left(k_{a}-\lambda_{2}\right) \cdot\left(\lambda_{1}-\lambda_{2}\right)} ; \\
& E_{4}=\frac{1}{\left(k_{a}-\lambda_{1}\right) \cdot\left(k_{a}-\lambda_{2}\right)} \\
& C_{2}(t)=\frac{x_{2}(t)}{V_{2}} \\
& x_{3}(t)=D \cdot \mathrm{e}^{-k_{a} \cdot t} \\
& C_{3}(t)=\frac{x_{3}(t)}{V_{3}}
\end{aligned}
$$

Partial sums and formulas of the mathematical Series for repetitive oral doses

$$
\begin{aligned}
C_{1, n, t}= & \frac{D \cdot k_{a}}{V_{1}} \cdot\left\{A_{3} \cdot \frac{\left(1-\mathrm{e}^{-\lambda_{1} \cdot n \cdot \tau}\right)}{\left(1-\mathrm{e}^{-\lambda_{1} \cdot \tau}\right)} \cdot \mathrm{e}^{-\lambda_{1} \cdot(t-(n-1) \cdot \tau)}\right. \\
& \left.+B_{3} \cdot \frac{\left(1-\mathrm{e}^{-\lambda_{2} \cdot n \cdot \tau}\right)}{\left(1-\mathrm{e}^{-\lambda_{2} \cdot \tau}\right)} \cdot \mathrm{e}^{-\lambda_{2} \cdot(t-(n-1) \cdot \tau)}+E_{3} \cdot \frac{\left(1-\mathrm{e}^{-k_{a} \cdot n \cdot \tau}\right)}{\left(1-\mathrm{e}^{-k_{a} \cdot \tau}\right)} \cdot \mathrm{e}^{-k_{a} \cdot(t-(n-1) \cdot \tau)}\right\} \\
C_{2, n, t}= & \frac{D \cdot k_{a} \cdot k_{12}}{V_{2}} \cdot\left\{A_{4} \cdot \frac{\left(1-\mathrm{e}^{-\lambda_{1} \cdot n \cdot \tau}\right)}{\left(1-\mathrm{e}^{-\lambda_{1} \cdot \tau}\right)} \cdot \mathrm{e}^{-\lambda_{1} \cdot(t-(n-1) \cdot \tau)}\right\}, t \in[0, \infty) \\
& \left.+B_{4} \cdot \frac{\left(1-\mathrm{e}^{-\lambda_{2} \cdot n \cdot \tau}\right)}{\left(1-\mathrm{e}^{-\lambda_{2} \cdot \tau}\right)} \cdot \mathrm{e}^{-\lambda_{2} \cdot(t-(n-1) \cdot \tau)}+E_{4} \cdot \frac{\left(1-\mathrm{e}^{-k_{a} \cdot n \cdot \tau}\right)}{\left(1-\mathrm{e}^{-k_{a} \cdot \tau}\right)} \cdot \mathrm{e}^{-k_{a} \cdot(t-(n-1) \cdot \tau)}\right\} n, t \in[0, \infty)
\end{aligned}
$$

The corresponding steady state concentrations of the drug in the two compartments are, 


$$
\begin{aligned}
C_{1, n, s s, t}= & \frac{D \cdot k_{a}}{V_{1}} \cdot\left\{\frac{A_{3}}{\left(1-\mathrm{e}^{-\lambda_{1} \cdot \tau}\right)} \cdot \mathrm{e}^{-\lambda_{1} \cdot(t-(n-1) \cdot \tau)}+\frac{B_{3}}{\left(1-\mathrm{e}^{-\lambda_{2} \cdot \tau}\right)} \cdot \mathrm{e}^{-\lambda_{2} \cdot(t-(n-1) \cdot \tau)}\right. \\
& \left.+\frac{E_{3}}{\left(1-\mathrm{e}^{-k_{a} \cdot \tau}\right)} \cdot \mathrm{e}^{-k_{a} \cdot(t-(n-1) \cdot \tau)}\right\} \\
C_{2, n, s s, t}= & \frac{D \cdot k_{a} \cdot k_{12}}{V_{2}} \cdot\left\{\frac{A_{4}}{\left(1-\mathrm{e}^{-\lambda_{1} \cdot \tau}\right)} \cdot \mathrm{e}^{-\lambda_{1} \cdot(t-(n-1) \cdot \tau)}+\frac{B_{4}}{\left(1-\mathrm{e}^{-\lambda_{2} \cdot \tau}\right)} \cdot \mathrm{e}^{-\lambda_{2} \cdot(t-(n-1) \cdot \tau)}\right. \\
& \left.+\frac{E_{4}}{\left(1-\mathrm{e}^{-k_{a} \cdot \tau}\right)} \cdot \mathrm{e}^{-k_{a} \cdot(t-(n-1) \cdot \tau)}\right\}
\end{aligned}
$$

Trough concentrations can be obtained from the general equations above at $t=n \cdot \tau$.

$$
\begin{aligned}
C_{1, n, \min }= & \frac{D \cdot k_{a}}{V_{1}} \cdot\left\{A_{3} \cdot \frac{\left(1-\mathrm{e}^{-\lambda_{1} \cdot n \cdot \tau}\right)}{\left(1-\mathrm{e}^{-\lambda_{1} \cdot \tau}\right)} \cdot \mathrm{e}^{-\lambda_{1} \cdot \tau}+B_{3} \cdot \frac{\left(1-\mathrm{e}^{-\lambda_{2} \cdot n \cdot \tau}\right)}{\left(1-\mathrm{e}^{-\lambda_{2} \cdot \tau}\right)} \cdot \mathrm{e}^{-\lambda_{2} \cdot \tau}\right. \\
+ & \left.E_{3} \cdot \frac{\left(1-\mathrm{e}^{-k_{a} \cdot n \cdot \tau}\right)}{\left(1-\mathrm{e}^{-k_{a} \cdot \tau}\right)} \cdot \mathrm{e}^{-k_{a} \cdot \tau}\right\} \in[0, \infty) \\
C_{2, n, \min }= & \frac{D \cdot k_{a} \cdot k_{12}}{V_{2}} \cdot\left\{A_{4} \cdot \frac{\left(1-\mathrm{e}^{-\lambda_{1} \cdot n \cdot \tau}\right)}{\left(1-\mathrm{e}^{-\lambda_{1} \cdot \tau}\right)} \cdot \mathrm{e}^{-\lambda_{1} \cdot \tau}+B_{4} \cdot \frac{\left(1-\mathrm{e}^{-\lambda_{2} \cdot n \cdot \tau}\right)}{\left(1-\mathrm{e}^{-\lambda_{2} \cdot \tau}\right)} \cdot \mathrm{e}^{-\lambda_{2} \cdot \tau}\right. \\
& \left.+E_{4} \cdot \frac{\left(1-\mathrm{e}^{-k_{a} \cdot n \cdot \tau}\right)}{\left(1-\mathrm{e}^{-k_{a} \cdot \tau}\right)} \cdot \mathrm{e}^{-k_{a} \cdot \tau}\right\} \\
C_{1, \min , s s}= & \frac{D \cdot k_{a}}{V_{1}} \cdot\left\{\frac{A_{3}}{\left(1-\mathrm{e}^{-\lambda_{1} \cdot \tau}\right)} \cdot \mathrm{e}^{-\lambda_{1} \cdot \tau}+\frac{B_{3}}{\left(1-\mathrm{e}^{-\lambda_{2} \cdot \tau}\right)} \cdot \mathrm{e}^{-\lambda_{2} \cdot \tau}+\frac{E_{3}}{\left(1-\mathrm{e}^{-k_{a} \cdot \tau}\right)} \cdot \mathrm{e}^{-k_{a} \cdot \tau}\right\} \\
C_{2, \min , s s}= & \frac{D \cdot k_{a} \cdot k_{12}}{V_{2}} \cdot\left\{\frac{A_{4}}{\left(1-\mathrm{e}^{-\lambda_{1} \cdot \tau}\right)} \cdot \mathrm{e}^{-\lambda_{1} \cdot \tau}+\frac{B_{4}}{\left(1-\mathrm{e}^{-\lambda_{2} \cdot \tau}\right)} \cdot \mathrm{e}^{-\lambda_{2} \cdot \tau}\right. \\
& \left.+\frac{E_{4}}{\left(1-\mathrm{e}^{-k_{a} \cdot \tau}\right)} \cdot \mathrm{e}^{-k_{a} \cdot \tau}\right\}
\end{aligned}
$$

AUC formulas

$$
\begin{gathered}
\mathrm{AUC}_{1, n, \tau}=\frac{D \cdot k_{a}}{V_{1}} \cdot\left[A_{3} \cdot \frac{\left(1-\mathrm{e}^{-\lambda_{1} \cdot n \cdot \tau}\right)}{\lambda_{1}}+B_{3} \cdot \frac{\left(1-\mathrm{e}^{-\lambda_{2} \cdot n \cdot \tau}\right)}{\lambda_{2}}+E_{3} \cdot \frac{\left(1-\mathrm{e}^{-k_{a} \cdot n \cdot \tau}\right)}{k_{a}}\right] \\
\mathrm{AUC}_{2, n, \tau}=\frac{D \cdot k_{a} \cdot k_{12}}{V_{2}} \cdot\left[A_{4} \cdot \frac{\left(1-\mathrm{e}^{-\lambda_{1} \cdot n \cdot \tau}\right)}{\lambda_{1}}+B_{4} \cdot \frac{\left(1-\mathrm{e}^{-\lambda_{2} \cdot n \cdot \tau}\right)}{\lambda_{2}}+E_{4} \cdot \frac{\left(1-\mathrm{e}^{-k_{a} \cdot n \cdot \tau}\right)}{k_{a}}\right] \\
\mathrm{AUC}_{1, \tau, s s}=\frac{D \cdot k_{a}}{V_{1}} \cdot\left[\frac{A_{3}}{\lambda_{1}}+\frac{B_{3}}{\lambda_{2}}+\frac{E_{3}}{k_{a}}\right] \\
\mathrm{AUC}_{2, \tau, s s}=\frac{D \cdot k_{a} \cdot k_{12}}{V_{2}} \cdot\left[\frac{A_{4}}{\lambda_{1}}+\frac{B_{4}}{\lambda_{2}}+\frac{E_{4}}{k_{a}}\right]
\end{gathered}
$$


The peak drug concentration in the central compartment can be obtained as a function of oral dose number after we obtain the time to reach maximum central compartment concentration by setting the derivatives in Equation (31) equal to zero.

The time to reach maximum drug concentration $t_{1, n \text {, max }}$, in the central compartment can be determined from Equation (49) by iteration.

$$
\begin{gathered}
\frac{\mathrm{d} x_{1, n, t}}{\mathrm{~d} t}=0 \\
\left\{\begin{array}{c}
-\lambda_{1} \cdot A_{3} \cdot \frac{\left(1-\mathrm{e}^{-\lambda_{1} \cdot n \cdot \tau}\right)}{\left(1-\mathrm{e}^{-\lambda_{1} \cdot \tau}\right)} \cdot \mathrm{e}^{-\lambda_{1} \cdot\left(t_{1, n, \max }-(n-1) \cdot \tau\right)}-\lambda_{2} \cdot B_{3} \cdot \frac{\left(1-\mathrm{e}^{-\lambda_{2} \cdot n \cdot \tau}\right)}{\left(1-\mathrm{e}^{-\lambda_{2} \cdot \tau}\right)} \cdot \mathrm{e}^{-\lambda_{2} \cdot\left(t_{1, n, \max }-(n-1) \cdot \tau\right)} \\
\left.-k_{a} \cdot E_{3} \cdot \frac{\left(1-\mathrm{e}^{-k_{a} \cdot n \cdot \tau}\right)}{\left(1-\mathrm{e}^{-k_{a} \cdot \tau}\right)} \cdot \mathrm{e}^{-k_{a} \cdot\left(t_{1, n, \max }-(n-1) \cdot \tau\right)}\right\}=0
\end{array}\right.
\end{gathered}
$$

A ballpark figure can be obtained if drug distribution is faster than drug absorption which is in turn much faster than drug elimination. Thus, if $\lambda_{1} \gg k_{a}$, $\mathrm{e}^{-\lambda_{1} \cdot\left(t_{1, n, \max }-(n-1) \cdot \tau\right)} \rightarrow 0$

$$
\begin{aligned}
& \mathrm{e}^{\left(k_{a}-\lambda_{2}\right) \cdot\left(t_{1, n, \max }-(n-1) \cdot \tau\right)}=-\frac{k_{a}}{\lambda_{2}} \cdot \frac{E_{3}}{B_{3}} \cdot \frac{\left(1-\mathrm{e}^{-k_{a} \cdot n \cdot \tau}\right)}{\left(1-\mathrm{e}^{-k_{a} \cdot \tau}\right)} \cdot \frac{\left(1-\mathrm{e}^{-\lambda_{2} \cdot \tau}\right)}{\left(1-\mathrm{e}^{-\lambda_{2} \cdot n \cdot \tau}\right)} \\
& \Rightarrow \\
& t_{1, n, \max }=(n-1) \cdot \tau+\frac{\ln \left(\frac{k_{a} \cdot\left(k_{21}-k_{a}\right) \cdot\left(\lambda_{2}-\lambda_{1}\right) \cdot\left(1-\mathrm{e}^{-k_{a} \cdot n \cdot \tau}\right) \cdot\left(1-\mathrm{e}^{-\lambda_{2} \cdot \tau}\right)}{\lambda_{2} \cdot\left(k_{a}-\lambda_{1}\right) \cdot\left(k_{21}-\lambda_{2}\right) \cdot\left(1-\mathrm{e}^{-k_{a} \cdot \tau}\right) \cdot\left(1-\mathrm{e}^{-\lambda_{2} \cdot n \cdot \tau}\right)}\right)}{\left(k_{a}-\lambda_{2}\right)} \\
& t_{1,1, \max }=\frac{\ln \left(\frac{k_{a} \cdot\left(k_{21}-k_{a}\right) \cdot\left(\lambda_{2}-\lambda_{1}\right)}{\lambda_{2} \cdot\left(k_{a}-\lambda_{1}\right) \cdot\left(k_{21}-\lambda_{2}\right)}\right)}{\left(k_{a}-\lambda_{2}\right)} \\
& t_{1, \max , s s}^{*}=\frac{\ln \left(\frac{k_{a} \cdot\left(k_{21}-k_{a}\right) \cdot\left(\lambda_{2}-\lambda_{1}\right) \cdot\left(1-\mathrm{e}^{-\lambda_{2} \cdot \tau}\right)}{\lambda_{2} \cdot\left(k_{a}-\lambda_{1}\right) \cdot\left(k_{21}-\lambda_{2}\right) \cdot\left(1-\mathrm{e}^{-k_{a} \cdot \tau}\right)}\right)}{\left(k_{a}-\lambda_{2}\right)} \\
& t_{1, \max , s s}^{*}=t_{1, \max , 1}+\frac{\ln \left(\frac{\left(1-\mathrm{e}^{-\lambda_{2} \cdot \tau}\right)}{\left(1-\mathrm{e}^{-k_{a} \cdot \tau}\right)}\right)}{\left(k_{a}-\lambda_{2}\right)} \\
& C_{1, n, \max }=\frac{D \cdot k_{a}}{V_{1}} \cdot\left\{A_{3} \cdot \frac{\left(1-\mathrm{e}^{-\lambda_{1} \cdot n \cdot \tau}\right)}{\left(1-\mathrm{e}^{-\lambda_{1} \cdot \tau}\right)} \cdot \mathrm{e}^{-\lambda_{1} \cdot\left(t_{1, n, \max }-(n-1) \cdot \tau\right)}\right. \\
& +B_{3} \cdot \frac{\left(1-\mathrm{e}^{-\lambda_{2} \cdot n \cdot \tau}\right)}{\left(1-\mathrm{e}^{-\lambda_{2} \cdot \tau}\right)} \cdot \mathrm{e}^{-\lambda_{2} \cdot\left(t_{1, n, \max }-(n-1) \cdot \tau\right)} \\
& \left.+E_{3} \cdot \frac{\left(1-\mathrm{e}^{-k_{a} \cdot n \cdot \tau}\right)}{\left(1-\mathrm{e}^{-k_{a} \cdot \tau}\right)} \cdot \mathrm{e}^{-k_{a} \cdot\left(t_{1, n, \max }-(n-1) \cdot \tau\right)}\right\}
\end{aligned}
$$




$$
C_{1, \max , s s}=\frac{D \cdot k_{a}}{V_{1}} \cdot\left\{A_{3} \cdot \frac{\mathrm{e}^{-\lambda_{1} \cdot t_{1, \max , s s}^{*}}}{\left(1-\mathrm{e}^{-\lambda_{1} \cdot \tau}\right)}+B_{3} \cdot \frac{\mathrm{e}^{-\lambda_{2} \cdot t_{1, \max , s s}^{*}}}{\left(1-\mathrm{e}^{-\lambda_{2} \cdot \tau}\right)}+E_{3} \cdot \frac{\mathrm{e}^{-k_{a} \cdot t_{1, \max , s s}^{*}}}{\left(1-\mathrm{e}^{-k_{a} \cdot \tau}\right)}\right\}
$$

The time to reach peak concentration $t_{2, \max , n}$, in the peripheral compartment can be determined by iteration from Equation (54), as shown below:

$$
\begin{aligned}
& \left\{A_{4} \cdot \frac{\left(1-\mathrm{e}^{-\lambda_{1} \cdot n \cdot \tau}\right)}{\left(1-\mathrm{e}^{-\lambda_{1} \cdot \tau}\right)} \cdot \mathrm{e}^{-\lambda_{1} \cdot(t-(n-1) \cdot \tau)}+B_{4} \cdot \frac{\left(1-\mathrm{e}^{-\lambda_{2} \cdot n \cdot \tau}\right)}{\left(1-\mathrm{e}^{-\lambda_{2} \cdot \tau}\right)} \cdot \mathrm{e}^{-\lambda_{2} \cdot(t-(n-1) \cdot \tau)}\right. \\
& \left.+E_{4} \cdot \frac{\left(1-\mathrm{e}^{-k_{a} \cdot n \cdot \tau}\right)}{\left(1-\mathrm{e}^{-k_{a} \cdot \tau}\right)} \cdot \mathrm{e}^{-k_{a} \cdot(t-(n-1) \cdot \tau)}\right\}=0
\end{aligned}
$$

We can obtain a ballpark estimate of $t_{2, n, \max }$ if $\lambda_{1} \gg k_{a}$.

$$
\begin{gathered}
\left\{\frac{\left(k_{21}-\lambda_{2}\right)}{\left(k_{a}-\lambda_{2}\right) \cdot\left(\lambda_{1}-\lambda_{2}\right)} \cdot \frac{\left(1-\mathrm{e}^{-\lambda_{2} \cdot n \cdot \tau}\right)}{\left(1-\mathrm{e}^{-\lambda_{2} \cdot \tau}\right)} \cdot \mathrm{e}^{-\lambda_{2} \cdot(t-(n-1) \cdot \tau)}\right. \\
\left.+\frac{\left(k_{21}-k_{a}\right)}{\left(k_{a}-\lambda_{1}\right) \cdot\left(k_{a}-\lambda_{2}\right)} \cdot \frac{\left(1-\mathrm{e}^{-k_{a} \cdot n \cdot \tau}\right)}{\left(1-\mathrm{e}^{-k_{a} \cdot \tau}\right)} \cdot \mathrm{e}^{-k_{a} \cdot(t-(n-1) \cdot \tau)}\right\}=0 \\
t_{2, n, \max }=(n-1) \cdot \tau+\frac{\ln \left(\frac{k_{a} \cdot\left(1-\mathrm{e}^{-k_{a} \cdot n \cdot \tau}\right) \cdot\left(\lambda_{2}-\lambda_{1}\right) \cdot\left(1-\mathrm{e}^{-\lambda_{2} \cdot \tau}\right)}{\lambda_{2} \cdot\left(1-\mathrm{e}^{-\lambda_{2} \cdot n \cdot \tau}\right) \cdot\left(k_{a}-\lambda_{1}\right) \cdot\left(1-\mathrm{e}^{-k_{a} \cdot \tau}\right)}\right)}{\left(k_{a}-\lambda_{2}\right)} \\
t_{2, \text { max }, s s}^{*}=\frac{\ln \left(\frac{k_{a} \cdot\left(\lambda_{2}-\lambda_{1}\right) \cdot\left(1-\mathrm{e}^{-\lambda_{2} \cdot \tau}\right)}{\lambda_{2} \cdot\left(k_{a}-\lambda_{1}\right) \cdot\left(1-\mathrm{e}^{-k_{a} \cdot \tau}\right)}\right)}{\left(k_{a}-\lambda_{2}\right)}
\end{gathered}
$$

Since at the time of peak peripheral concentration the intercompartmental solute exchange rates are equal, $t_{2, n, \max }=t_{e q, n}$, where $t_{e q, n}$ is the time of momentary distribution equilibrium as a function of dose number $(n)$. The time of momentary distribution equilibrium during the first dosing interval $(n=1)$ is,

$$
t_{e q, 1}=t_{2,1, \max }=\frac{\ln \left[\frac{k_{a} \cdot\left(\lambda_{2}-\lambda_{1}\right)}{\lambda_{2} \cdot\left(k_{a}-\lambda_{1}\right)}\right]}{\left(k_{a}-\lambda_{2}\right)}
$$

The non-steady state and steady state peak peripheral drug concentration and central compartment drug concentration are expressed with Equations (57)(60).

$$
\begin{aligned}
C_{2, n, \text { max }}= & \frac{D \cdot k_{a} \cdot k_{12}}{V_{2}} \cdot\left\{A_{4} \cdot \frac{\left(1-\mathrm{e}^{-\lambda_{1} \cdot n \cdot \tau}\right)}{\left(1-\mathrm{e}^{-\lambda_{1} \cdot \tau}\right)} \cdot \mathrm{e}^{-\lambda_{1} \cdot\left(t_{2, n, \max }-(n-1) \cdot \tau\right)}\right. \\
& +B_{4} \cdot \frac{\left(1-\mathrm{e}^{-\lambda_{2} \cdot n \cdot \tau}\right)}{\left(1-\mathrm{e}^{-\lambda_{2} \cdot \tau}\right)} \cdot \mathrm{e}^{-\lambda_{2} \cdot\left(t_{2, n, \max }-(n-1) \cdot \tau\right)} \\
& \left.+E_{4} \cdot \frac{\left(1-\mathrm{e}^{-k_{a} \cdot n \cdot \tau}\right)}{\left(1-\mathrm{e}^{-k_{a} \cdot \tau}\right)} \cdot \mathrm{e}^{-k_{a} \cdot\left(t_{2, n, \text { max }}-(n-1) \cdot \tau\right)}\right\}
\end{aligned}
$$




$$
\begin{aligned}
& C_{2, \max , s s}=\frac{D \cdot k_{a} \cdot k_{12}}{V_{2}} \cdot\left\{A_{4} \cdot \frac{\mathrm{e}^{-\lambda_{1} \cdot t_{2, \max , s s}^{*}}}{\left(1-\mathrm{e}^{-\lambda_{1} \cdot \tau}\right)}+B_{4} \cdot \frac{\mathrm{e}^{-\lambda_{2} \cdot t_{2, \max , s s}^{*}}}{\left(1-\mathrm{e}^{-\lambda_{2} \cdot \tau}\right)}+E_{4} \cdot \frac{\mathrm{e}^{-k_{a} \cdot t_{2, \max , s s}^{*}}}{\left(1-\mathrm{e}^{-k_{a} \cdot \tau}\right)}\right\} \\
& C_{1, e q, n}=\frac{D \cdot k_{a}}{V_{1}} \cdot\left\{A_{3} \cdot \frac{\left(1-\mathrm{e}^{-\lambda_{1} \cdot n \cdot \tau}\right)}{\left(1-\mathrm{e}^{-\lambda_{1} \cdot \tau}\right)} \cdot \mathrm{e}^{-\lambda_{1} \cdot\left(t_{2, n, \max }-(n-1) \cdot \tau\right)}\right. \\
& +B_{3} \cdot \frac{\left(1-\mathrm{e}^{-\lambda_{2} \cdot n \cdot \tau}\right)}{\left(1-\mathrm{e}^{-\lambda_{2} \cdot \tau}\right)} \cdot \mathrm{e}^{-\lambda_{2} \cdot\left(t_{2, n, \max }-(n-1) \cdot \tau\right)} \\
& \left.+E_{3} \cdot \frac{\left(1-\mathrm{e}^{-k_{a} \cdot n \cdot \tau}\right)}{\left(1-\mathrm{e}^{-k_{a} \cdot \tau}\right)} \cdot \mathrm{e}^{-k_{a} \cdot\left(t_{2, n, \max }-(n-1) \cdot \tau\right)}\right\} \\
& C_{1, e q, s s}=\frac{D \cdot k_{a}}{V_{1}} \cdot\left\{A_{3} \cdot \frac{1}{\left(1-\mathrm{e}^{-\lambda_{1} \cdot \tau}\right)} \cdot \mathrm{e}^{-\lambda_{1} \cdot t_{2, \text { max }, s s}^{*}}+B_{3} \cdot \frac{1}{\left(1-\mathrm{e}^{-\lambda_{2} \cdot \tau}\right)} \cdot \mathrm{e}^{-\lambda_{2} \cdot t_{2, \text { max }, s s}^{*}}\right. \\
& \left.+E_{3} \cdot \frac{1}{\left(1-\mathrm{e}^{-k_{a} \cdot \tau}\right)} \cdot \mathrm{e}^{-k_{a} \cdot t_{2, \max , s s}^{*}}\right\}
\end{aligned}
$$

\subsection{Pharmacokinetic Analysis}

Prazosin plasma drug concentration-time values were extracted from Figure 1 of the published work of Bateman and coworkers (single short intravenous infusion and single oral dose) and the work of Grahnen et al., using the online graph reader tool (graphreader.com). The correctness of the extracted C-t values was also verified by manually drawn values on scaled log y-axis. All pharmacokinetic parameters were determined using linear and nonlinear regression analysis of plasma drug concentration-time data.

\subsection{Computer Simulations}

Simulations of drug concentration and AUC as a function of time were carried out with Microsoft Excel Spreadsheet Software [3] [14] [15].

\section{Results}

\subsection{Single IV Bolus}

Simple linear regression analysis was carried out on published plasma drug concentration as a function of time on four patients using the least squares method [6]. In accord with the two-compartment open model, the plasma is part of the central compartment. Initial estimates of model parameters were obtained by the method of residuals where the coefficient $\left(\frac{D}{V_{1}} \cdot B_{1}\right)$ and eigenvector $\lambda_{2}$ were determined from the intercept and slope of the regressed best-fit logarithmic equation of measured plasma drug concentration as a function of terminal time data points where drug distribution reached steady-state, respectively. These two fitted parameters were then used to calculate the drug concentration that was removed from the plasma purely due to drug elimination $\left(C_{1}^{\prime}\right)$. The residual was 
calculated from the difference between the measured plasma drug concentration $\left(C_{1}\right)$ and $C_{1}^{\prime}$. A plot of the logarithmic concentration difference $\left(C_{1}-C_{1}^{\prime}\right)$ with time at early data points was linear. The slope and intercept of the best-fit equation were used to determine the eigenvector $\lambda_{1}$ and coefficient $\left(\frac{D}{V_{1}} \cdot A_{1}\right)$, respectively. The number of data points that were used to assess the four parameters was decided from the correlation coefficient of each line and the contribution of the two eigenvectors on each phase. In general, the contribution of the distribution phase as assessed from the exponential ratio of the two eigenvectors $\mathrm{e}^{-\left(\lambda_{1}+\lambda_{2}\right) \cdot t}$ to the terminal time data points was between $5.66 \times 10^{-10} \%$ and $0.16 \%$. In agreement with these results of the eigenvector exponential ratio, the contribution of the distribution process to the plasma drug concentration calculated using the first-order formula $\left(\frac{1}{2}\right)^{\left(\frac{t}{t_{1 / 2, \alpha}}\right)}$ was also found to be zero for the terminal data.

The average estimates out of four patients of the aforementioned parameters were utilized to obtain an initial estimate of the volume of the central compartment. The volume of the central compartment was determined from the initial concentration of the drug in the central compartment after the first intravenous dose $C_{1,1,0}$ using Equation (9a). Initial estimates of the unitless coefficients $A_{1}$ and $B_{1}$ were calculated from the administered dose and the $V_{1}$. Further optimization was carried out using the Excel GRG Nonlinear method of iteration by varying simultaneously all five parameters $V_{1}, A_{1}, B_{1}, \lambda_{1}$ and $\lambda_{2}$. The average value of $V_{1}$ was calculated to be $11.25 \mathrm{~L}$. Finally, the values of the microrate intercompartmental constants, $k_{21}, k_{10}$ and $k_{12}$ were calculated using Equations (61)-(63) (Table 1).

$$
\begin{gathered}
k_{21}=\frac{A_{1} \cdot \lambda_{2}+B_{1} \cdot \lambda_{1}}{A_{1}+B_{1}} \\
k_{10}=\frac{\lambda_{1} \cdot \lambda_{2}}{k_{21}} \\
k_{12}=\lambda_{1}+\lambda_{2}-k_{10}-k_{21}
\end{gathered}
$$

Since the volume of the central compartment is less than the extracellular body fluid we can reasonably assume that the central compartment is a monophase and use Riggs equation (Equation (64)) to determine the apparent volume of distribution of the drug with respect to the central phase [16].

$$
V_{d, 1}=V_{1} \cdot\left(1+\frac{k_{12}}{k_{21}}\right)
$$

The average value of $V_{d, 1}$ was found to be equal to $32.87 \mathrm{~L}$. Since this value is not greater than the total body fluid of a $70-\mathrm{kg}$ healthy human subject, and the drug is not known to be involved with any influx transport system we can reasonably assume that $V_{d, 1}$ is not the apparent but it is the actual volume of distribution of the drug in the body. The volume of peripheral compartment can be 
Table 1. Pharmacokinetic parameters of Prazosin after a single $0.5 \mathrm{mg}$ intravenous bolus dose in a two-compartment open model.

\begin{tabular}{|c|c|c|c|c|c|}
\hline \multicolumn{6}{|c|}{ Patient } \\
\hline parameter & B. G. & E. $R$. & H. A. & M. F. & Average \pm St Dev \\
\hline$W(\mathrm{~kg})$ & 85 & 65 & 110 & 76 & $84 \pm 19.2$ \\
\hline$\star \alpha \quad\left(\mathrm{h}^{-1}\right)$ & 4.0073 & 4.8501 & 3.5422 & 7.8375 & $5.0593 \pm 1.9296$ \\
\hline$\star \beta \quad\left(\mathrm{h}^{-1}\right)$ & 0.2613 & 0.3327 & 0.1968 & 0.2911 & $0.2705 \pm 0.0572$ \\
\hline$k_{12} \quad\left(\mathrm{~h}^{-1}\right)$ & 2.1958 & 2.6317 & 1.7450 & 5.1062 & $2.9197 \pm 1.5020$ \\
\hline$k_{21}\left(\mathrm{~h}^{-1}\right)$ & 1.2003 & 1.1591 & 1.5420 & 1.4663 & $1.3419 \pm 0.1906$ \\
\hline$k_{10} \quad\left(\mathrm{~h}^{-1}\right)$ & 0.8725 & 1.3919 & 0.4520 & 1.5561 & $1.0681 \pm 0.5036$ \\
\hline$V_{1} \quad(\mathrm{~L})$ & 9.00 & 8.84 & 18.96 & 8.18 & $11.25 \pm 5.16$ \\
\hline$V_{2} \quad(\mathrm{~L})$ & 16.46 & 20.07 & 21.46 & 28.50 & $21.62 \pm 5.04$ \\
\hline$V_{d, 1} \quad(\mathrm{~L})$ & 25.46 & 28.91 & 40.42 & 36.68 & $32.87 \pm 6.88$ \\
\hline $\mathrm{AUC}_{\mathrm{ss}}(\mathrm{ng} \cdot \mathrm{h} / \mathrm{mL})$ & 63.68 & 40.63 & 58.33 & 39.26 & $50.48 \pm 12.37$ \\
\hline $\mathrm{CL}(\mathrm{L} / \mathrm{h})$ & 7.85 & 12.31 & 8.57 & 12.73 & $10.37 \pm 2.51$ \\
\hline$A_{1}$ & 0.7493 & 0.8170 & 0.5979 & 0.8443 & $0.7521 \pm 0.1103$ \\
\hline$B_{1}$ & 0.2507 & 0.1830 & 0.4021 & 0.1557 & $0.2479 \pm 0.1103$ \\
\hline$A_{2} \quad\left(\mathrm{~h}^{-1}\right)$ & -0.2670 & -0.2214 & -0.2989 & -0.1325 & $-0.2299 \pm 0.0723$ \\
\hline$B_{2} \quad\left(\mathrm{~h}^{-1}\right)$ & 0.2670 & 0.2214 & 0.2989 & 0.1325 & $0.2299 \pm 0.0723$ \\
\hline
\end{tabular}

determined from the difference of the two volumes. Its value is close to intracellular body fluid volume.

$$
V_{2}=V_{d, 1}-V_{1}=21.62 \mathrm{~L}
$$

\subsection{Single Intermittent Intravenous Infusion}

One of the most comprehensive pharmacokinetic studies of prazosin was conducted by Bateman and coworkers. Although the drug was administered by a slow intravenous 10-minute infusion the two-compartment model average pharmacokinetic parameters were calculated with an IV bolus model using a mid-point rule technique, that is, they have timed sampling not from zero time but from the mid-point of the infusion. This was necessary as the intermittent IV infusion analytical solutions to drug concentration, especially during the infusion phase of the drug, were not available or because nonlinear regression analysis was difficult at the time. We have recently produced the explicit solutions to drug concentration in the one- and two-compartment models after multiple constant rate intermittent intravenous infusions for both ascending and descending phases [3] [14]. First, we time-shifted their sampling times by $5 \mathrm{mi}$ nutes, that is, the time 10 and 20 minutes became the actual time of 15 and 25 minutes, respectively, and so on and so forth. Second, the plasma drug concentration-time data points after administration of the first dose were fitted using non-linear least squares analysis by the intermittent infusion elimination phase 
equations (Equations (65)-(67)) in a two-compartment model [3]. The plot in Figure 2 shows the actual data points extracted from Bateman et al., and our model's fitted line. Notice that with our method we can also recover the peak drug concentration at the end of the infusion period. All calculated average pharmacokinetic parameters of Prazosin are listed in Table 2.

$$
\begin{aligned}
C_{e, 1,1}(t)= & \frac{\left(k_{21}-\lambda_{1}\right) \cdot C_{1}(T)+k_{21} \cdot C_{2}(T)}{\left(\lambda_{2}-\lambda_{1}\right)} \cdot \mathrm{e}^{-\lambda_{1} \cdot(t-T)} \\
& +\frac{\left(k_{21}-\lambda_{2}\right) \cdot C_{1}(T)+k_{21} \cdot C_{2}(T)}{\left(\lambda_{1}-\lambda_{2}\right)} \cdot \mathrm{e}^{-\lambda_{2} \cdot(t-T)}
\end{aligned}
$$

where,

$$
\begin{gathered}
C_{1}(T)=\frac{k_{0}}{V_{1}} \cdot\left(\frac{k_{21}}{\lambda_{1} \cdot \lambda_{2}}+\frac{k_{21}-\lambda_{1}}{\lambda_{1} \cdot\left(\lambda_{1}-\lambda_{2}\right)} \cdot \mathrm{e}^{-\lambda_{1} \cdot T}+\frac{k_{21}-\lambda_{2}}{\lambda_{2} \cdot\left(\lambda_{2}-\lambda_{1}\right)} \cdot \mathrm{e}^{-\lambda_{2} \cdot T}\right) \\
C_{2}(T)=\frac{k_{0} \cdot k_{12}}{V_{2}} \cdot\left(\frac{1}{\lambda_{1} \cdot \lambda_{2}}+\frac{1}{\lambda_{1} \cdot\left(\lambda_{1}-\lambda_{2}\right)} \cdot \mathrm{e}^{-\lambda_{1} \cdot T}+\frac{1}{\lambda_{2} \cdot\left(\lambda_{2}-\lambda_{1}\right)} \cdot \mathrm{e}^{-\lambda_{2} \cdot T}\right)
\end{gathered}
$$

\subsection{Single $1 \mathrm{mg}$ Prazosin Oral Tablet Administration}

The average pharmacokinetic parameters of Prazosin in a two-compartment model were determined from measured plasma drug concentration in six patients (Bateman and coworkers) after administration of a single $1 \mathrm{mg}$ Prazosin tablet (Table 3). The C-t pairs were regressed in the two-compartment model using Equation (34) by minimizing the sum of the squares of the residuals. Regression analysis of the data points was also carried out in the one-compartment model using Equation (68). Neither the two-compartment model nor the onecompartment model fitted the average plasma drug concentrations of the absorption phase very well (Figure 3 ).

$$
C=\frac{F \cdot D \cdot k_{a}}{V_{d} \cdot\left(k_{a}-k\right)} \cdot\left(\mathrm{e}^{-k \cdot t}-\mathrm{e}^{-k_{a} \cdot t}\right)
$$

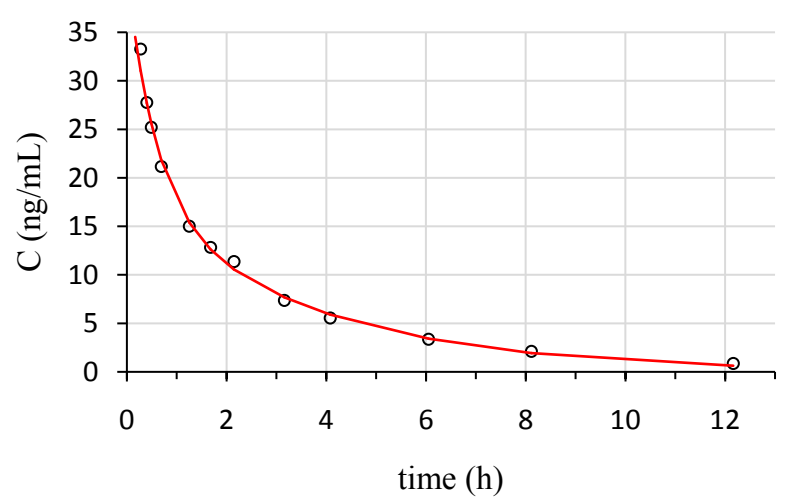

Figure 2. (empty circles, $\circ$ ) Measured plasma Prazosin concentration (data extracted from the published work of Bateman et al.,) after a single 10-minute constant rate infusion of Prazosin $1 \mathrm{mg}$. (solid line) Model's fitted line using Equation (65). 
Table 2. Average pharmacokinetic parameters after administration of a single intermittent intravenous infusion of $1 \mathrm{mg}$ Prazosin at a rate of $0.1 \mathrm{mg} / \mathrm{min}$. Plasma prazosin concentration-time points were extracted from the article of Bateman and coworkers.

\begin{tabular}{|c|c|c|c|c|c|c|c|}
\hline$\lambda_{1} \quad\left(\mathrm{~h}^{-1}\right)$ & $\lambda_{2} \quad\left(\mathrm{~h}^{-1}\right)$ & $k_{12} \quad\left(\mathrm{~h}^{-1}\right)$ & $k_{21}\left(\mathrm{~h}^{-1}\right)$ & $k_{10} \quad\left(\mathrm{~h}^{-1}\right)$ & $V_{1} \quad(\mathrm{~L})$ & $V_{2} \quad(\mathrm{~L})$ & $V_{d, 1} \quad(\mathrm{~L})$ \\
\hline 1.76 & 0.28 & 0.5813 & 0.9373 & 0.5179 & 26.5 & 14.2 & 42.9 \\
\hline
\end{tabular}

Table 3. Average pharmacokinetic parameters after administration of a single $1 \mathrm{mg}$ Prazosin oral tablet. Plasma prazosin concentration-time data points were extracted from the published article of Bateman and coworkers.

\begin{tabular}{cccc}
\hline \multicolumn{2}{c}{ Parameter } & One-Compartment & Two-Compartment \\
\hline$\lambda_{1}$ & $\left(\mathrm{~h}^{-1}\right)$ & --- & 3.1761 \\
$*$ & $\lambda_{2}\left(\mathrm{~h}^{-1}\right)$ & 0.3171 & 0.3171 \\
$k_{12}$ & $\left(\mathrm{~h}^{-1}\right)$ & --- & 1.1451 \\
$k_{21}$ & $\left(\mathrm{~h}^{-1}\right)$ & --- & 1.7834 \\
$k_{10}$ & $\left(\mathrm{~h}^{-1}\right)$ & --- & 0.5645 \\
$k_{a}$ & $\left(\mathrm{~h}^{-1}\right)$ & 0.6957 & 0.5335 \\
$V_{1}$ & 0.547 & 0.614 \\
$V_{2}$ & $(\mathrm{~L})$ & --- & 26.5 \\
$V_{d, 1}$ & $(\mathrm{~L})$ & --- & 17.0 \\
\hline
\end{tabular}

* $\lambda_{2}=k$ (for the one-compartment model).

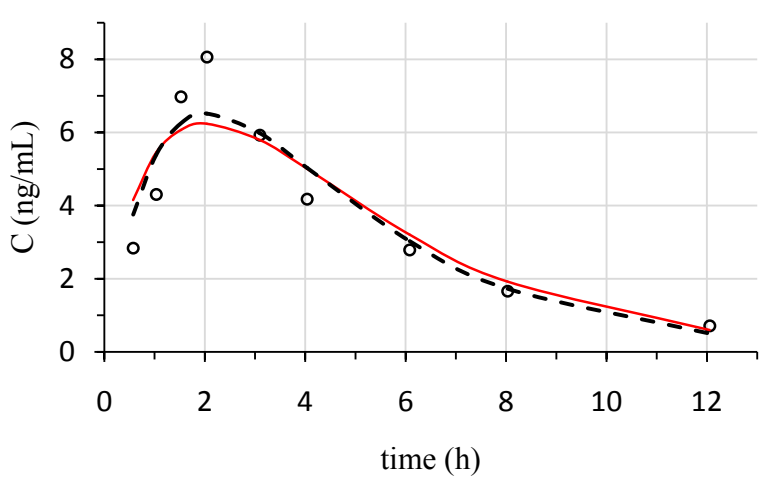

Figure 3. The average plasma Prazosin concentration (measured concentrations were extracted from Bateman et al., empty symbols) was simulated as a function of time by a one-compartment model (dashed-line) and a two-compartment model (solid line).

\section{Discussion}

\subsection{Single IV Bolus}

The estimated pharmacokinetic parameters of Prazosin in Table 1 are very similar to those published by Grahnen and coworkers. Using these parameters that were extracted from a single IV bolus we were able to simulate the Prazosin concentration in the central and peripheral compartment after repetitive intravenous boluses at constant dosing frequency as a function of total time using 
Equation (9) and Equation (10), respectively. As shown in Figure 4, the plasma drug concentration in the central compartment fluctuates from about $53 \mathrm{ng} / \mathrm{mL}$ to $9 \mathrm{ng} / \mathrm{mL}$. The time to reach peak peripheral drug concentration, calculated with Equation (23) and Equation (26) at non-steady state and steady state conditions, respectively, varied with the IV bolus dose number ( $n$ ) from 36.7 minutes to 29.3 minutes (Table 4).

The apparent volume of distribution of a drug in a two-compartment model was calculated using Equation (64). If our assumption is correct about the central monophase compartment, we should be able to get a similar value for the apparent volume of distribution of Prazosin associated with the central compartment $V_{d, 1}$, using Equation (69) [2]. As we have discussed extensively elsewhere, the apparent volume of distribution is a system equilibrium property and

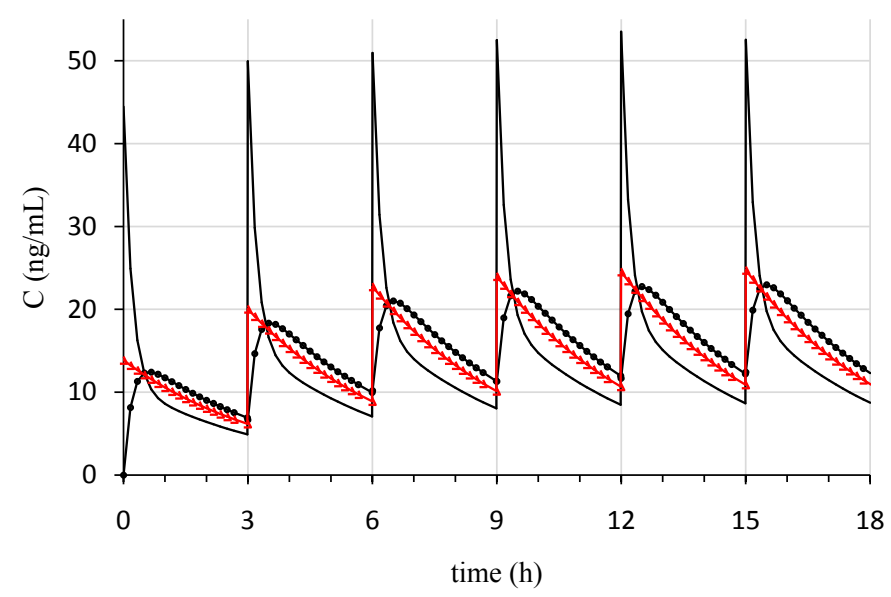

Figure 4. Simulated Prazosin concentration in the central compartment or plasma (solid line) and in the peripheral compartment (empty circles joined by solid line) as a function of time based on a twocompartment pharmacokinetic model. For comparison the simulated plasma drug concentration from a one-compartment model is also drawn (empty triangles joined with solid line). The dosing interval in all simulations was kept constant ( $\tau=3 \mathrm{~h}$ ).

Table 4. AUC (ng/mL.h) after repetitive $0.5 \mathrm{mg}$ Prazosin intravenous boluses at the end of each dosing interval ( $\tau=3 \mathrm{~h}$ ) in the one- and two-compartment models, and time to reach peak peripheral drug concentration $\left(t_{2, \text { max } x}^{*}\right)$, in units of minutes, as a function of dose number.

\begin{tabular}{ccccc}
\hline$n$ & $\mathrm{AUC}_{\text {one comp }}$ & $\mathrm{AUC}_{1, \max }$ & $\mathrm{AUC}_{2, \max }$ & $t_{2, \max , n}^{*}$ \\
\hline 1 & 28.5 & 29.3 & 28.8 & 36.7 \\
2 & 41.2 & 39.3 & 43.0 & 32.1 \\
3 & 46.8 & 43.8 & 49.3 & 30.5 \\
4 & 49.3 & 45.8 & 52.1 & 29.8 \\
5 & 50.4 & 46.6 & 53.3 & 29.6 \\
6 & 50.9 & 47.0 & 53.9 & 29.4 \\
Steady state & 51.3 & 47.3 & 54.3 & 29.3 \\
\hline
\end{tabular}


can be determined at times of momentary distribution equilibrium, i.e., when the rates of distribution of Prazosin molecules from central to peripheral compartment and vice versa are equal [3]. The value of $V_{d, 1}$ as calculated from the slope of the line in Figure 5 was $36.06 \mathrm{~L}$ which is within the average range of the $V_{d, 1}$ calculated with Equation (64).

$$
\begin{gathered}
V_{d, 1}=\frac{x_{1, e q, n}+x_{2, e q, n}}{C_{1, e q, n}}=\frac{x_{s, e q, n}}{C_{1, e q, n}} \\
C_{\text {one-comp }, n, t}=\frac{D}{V_{d, 1}} \cdot \frac{\left(1-\mathrm{e}^{-k \cdot n \cdot \tau}\right)}{\left(1-\mathrm{e}^{-k \cdot \tau}\right)} \cdot \mathrm{e}^{-k \cdot(t-(n-1) \cdot \tau)} \\
C_{\text {one-comp }, s s}=\frac{D}{V_{d, 1}} \cdot \frac{1}{\left(1-\mathrm{e}^{-k \cdot \tau}\right)} \cdot \mathrm{e}^{-k \cdot(t-(n-1) \cdot \tau)} \\
\mathrm{AUC}_{\text {one-comp }, \tau, n}=\frac{D}{k \cdot V_{d, 1}} \cdot\left(1-\mathrm{e}^{-k \cdot n \cdot \tau}\right) \\
\mathrm{AUC}_{1, \tau, s s}=\frac{D}{k \cdot V_{d, 1}}
\end{gathered}
$$

Obtaining the value of $V_{d, 1}$ is extremely important because it is the only way to carry out simulations of drug concentration with time and calculate the AUC after repetitive IV boluses in the one-compartment model. Equations (70)-(73) simulate non-steady state and steady state drug concentrations and AUC in the one-compartment model. As shown in Figure 4, the peak drug concentration after the first IV bolus using the same dose in the one-compartment model is around $14 \mathrm{ng} / \mathrm{mL}$ which is about one third of the value calculated from a two-compartment model $(\sim 44.5 \mathrm{ng} / \mathrm{mL})$. Although the concentration falls very

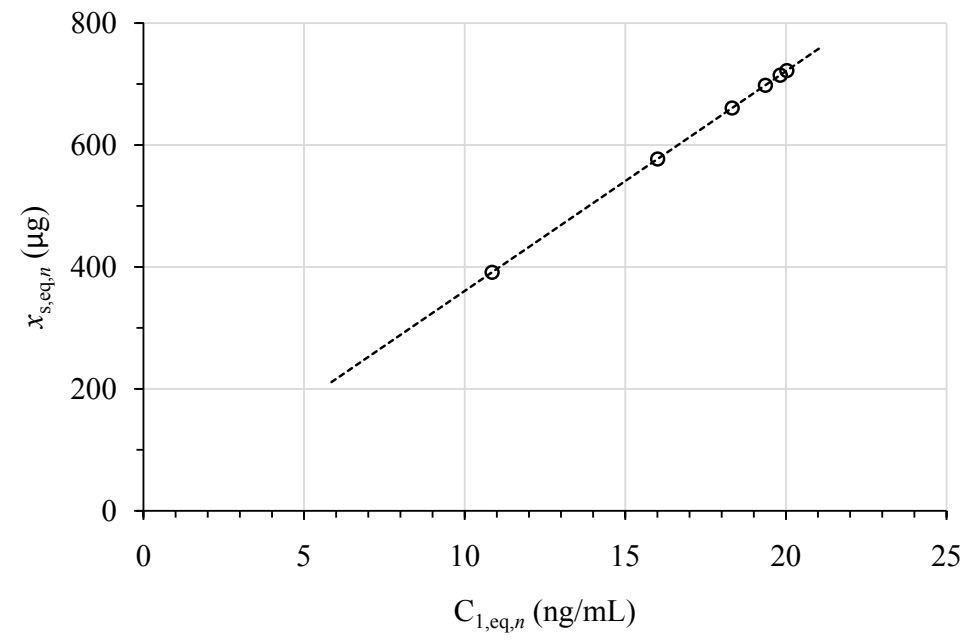

Figure 5. The amount of drug in the body $x_{s, e q, n}$ after repetitive intravenous boluses, was plotted against the drug concentration in the central compartment at momentary distribution equilibrium $C_{1, e q, n}$ as a function of dose number. The Prazosin apparent volume of distribution $\left(V_{d, 1}\right)$ was calculated from the slope of the line using Equation (69) to be equal to $36.06 \mathrm{~L}$. 
rapidly due to the very short distribution half-life, this could be significant if the drug concentration in the central compartment is as important as the drug concentration in the peripheral compartment for pharmacological activity. Prazosin is known to be a selective postsynaptic $\alpha_{1}$-adrenoceptor blocker. Its blood pressure (BP) lowering effect is exerted after binding and inhibiting $\alpha_{1}$-adrenoceptors in the vascular smooth muscle of mainly arteries and arterioles which from the pharmacokinetic viewpoint these tissues are in the central compartment [17] [18]. Furthermore, the inhibitory effect of Prazosin must be related to its binding affinity on these receptors, which is in turn concentration dependent [4]. It is therefore highly possible that the peak plasma concentration of Prazosin is the one that drives a higher receptor occupancy with a more pronounced antihypertensive effect. The concentration of Prazosin in the peripheral compartment is important in maintaining but not initiating its pharmacological effect. If this is the case then although the drug has a distribution half-life of only about $10 \mathrm{mi}$ nutes as compared to an elimination half-life of about $2.4 \mathrm{~h}$, the one-compartment model will not be able to approximate dosing calculations in the clinic. In support of this conclusion, Bateman et al., recorded highest drop in standing blood pressure during the first one half hour after an IV bolus administration of Prazosin. The one-compartment model simulated steady state peak prazosin plasma concentration is about one half of the corresponding two-compartment model estimate (Figure 4). It is not known if the reduction in blood pressure occurs immediately after Prazosin injection as the earliest sample was taken 30 minutes after IV Prazosin administration [5] [6]. Furthermore, the maximum reduction in BP found after 30 minutes coincides with the time to reach maximum peripheral concentration, $t_{2, \text { max }, n}^{*}$ after an IV bolus administration (Table 4). The time to reach maximum peripheral drug concentration after an intermittent infusion was about 1 hour. Thus, it is not possible to resolve from these studies the role of each compartment on the timings of the pharmacological activity of the drug. Similarly, the drop in standing blood pressure is correlated with the peak Prazosin plasma concentration, that is, during the first 2 - 4 hours after an oral drug administration [5] [10] [13] [19] [20]. According to these studies, the minimum effective plasma concentration is about $6 \mathrm{ng} / \mathrm{mL}$ while a marginal reduction of mean arterial pressure was reported by Horowitz and coworkers at peak concentration of $4.1 \pm 1.4 \mathrm{ng} / \mathrm{mL}$.

The body's exposure to the drug was assessed from the AUC that was calculated in the one- and two-compartment models at non-steady state and steady state conditions using Equation (72) and Equations (17)-(18), and Equation (73) and Equations (19)-(20), respectively (Table 4). Grahnen and coworkers have reported an AUC after a $0.5 \mathrm{mg}$ single prazosin IV dose of $49.4 \pm 12$ as calculated by the trapezoidal method plus the extrapolated area beyond the last sampling point which was always less than $15 \%$ of the total area. The AUCs in Table 4 are calculated for a 3-h dosing interval. Changing our dosing interval to $12 \mathrm{~h}$ results in a central compartment area $\mathrm{AUC}_{1, \tau=12 \mathrm{~h}}$ of $45.8 \mathrm{ng} / \mathrm{mL} \cdot \mathrm{h}$, which is almost identical to their reported experimentally measured average value. 
In his excellent work, Gibaldi has concluded that after intravenous bolus injection the drug distribution ratio in the two compartments in the $\beta$-phase is constant (Equation (74)) [21]. Using Equation (74), the prazosin tissue to central compartment drug mass ratio is equal to 2.72 .

$$
\left(\frac{x_{2}}{x_{1}}\right)_{\beta}=\frac{k_{12}}{k_{21}-\beta}
$$

We can assess the drug ratio in the two compartments using the non steady-state and the steady-state Equations (9)-(12).

$$
\left(\frac{x_{2, s s, t^{*}}}{x_{1, s s, t^{*}}}\right)=\frac{-k_{12} \cdot\left(\left(1-\mathrm{e}^{-\lambda_{2} \cdot \tau}\right) \cdot \mathrm{e}^{-\lambda_{1} \cdot t^{*}}-\left(1-\mathrm{e}^{-\lambda_{1} \cdot \tau}\right) \cdot \mathrm{e}^{-\lambda_{2} \cdot t^{*}}\right)}{\left(\left(\lambda_{1}-k_{21}\right) \cdot\left(1-\mathrm{e}^{-\lambda_{2} \cdot \tau}\right) \cdot \mathrm{e}^{-\lambda_{1} \cdot t^{*}}+\left(k_{21}-\lambda_{2}\right) \cdot\left(1-\mathrm{e}^{-\lambda_{1} \cdot \tau}\right) \cdot \mathrm{e}^{-\lambda_{2} \cdot t^{*}}\right)}
$$

The necessary condition for Equation (75) to be approximated by Equation (74) is for $\lambda_{1} \gg \lambda_{2}$ but Equation (75) can tell us much more about the time within a dosing interval that the drug distribution ratio will reach the constant value as dictated by Equation (74). Riegelman et al., have calculated the values $0.08483,01233,02670$ and 0.04930 for $k_{12}, k_{21}, \lambda_{1}$ and $\lambda_{2}$, respectively, for acetylsalicylic acid [22]. It turns out that for a dosing frequency $\tau=6 \mathrm{~h}$ the drug distribution ratio will never reach a constant value within the dosing interval. At the end of the dosing interval the drug ratio is only $76 \%$ of the final value and it takes about 9 hours from the time of administration for the drug distribution ratio to reach the value of Equation (74) within the $\beta$-elimination phase.

\subsection{Single Intermittent Intravenous Infusion}

The average pharmacokinetic parameters in Table 2 differ significantly from the ones reported by Bateman et al., with the exception of the elimination rate constant $\beta$ or $\lambda_{2}$. The trend in the two published IV bolus and intermittent infusion studies (and in our study) regarding the values of macro rate constants is similar, i.e. $\lambda_{1}>\lambda_{2}$ but not of the micro rate constants $k_{12}, k_{21}$ and $k_{10}$. The differences in our parameter estimation were due to first, we have used a two-compartment intermittent infusion model as opposed to the IV bolus model that they have used and second, we have fitted all data points to our model (Figure 2). It is possible that Bateman and coworkers have used only the first one or two data points to assess the value of the $\lambda_{1}$ hybrid rate constant for drug distribution and the last four data points for the $\lambda_{2}$ hybrid rate constant for drug elimination. As a result, the measured drug plasma concentrations after $1,1.5$ and 2 hours are not very close to the fitted line in Bateman's work. What is more interesting from our analysis is that although the apparent volume of distribution of Prazosin $V_{d, 1}$ is almost equal to total body water in both IV bolus and intermittent infusion studies, the trend in the volumes of the central and peripheral compartment is reversed. In particular, from the IV bolus study, $V_{1}$ and $V_{2}$ are $11.3 \mathrm{~L}$ and $21.6 \mathrm{~L}$, respectively, whereas the two volumes $V_{1}$ and $V_{2}$ that we have assessed from the intermittent drug infusion study are $26.5 \mathrm{~L}$ and 14.2 L, respectively. Bateman and coworkers have also estimated a larger 
central than peripheral compartment volume. Thus, slower drug infusion resulted in a larger volume of the central compartment at the expense of the volume of the peripheral compartment. It is possible that the kinetics of drug administration controls the volumes of the two compartments. And it could be that drug disposition after administering the drug at a very slow infusion rate be described by a one-compartment model. As we have cautioned in our recent publication, the kinetics of drug disposition after the first dose may not be identical after the second or third drug doses [14]. Incidentally, Grahnen et al. have not determined the apparent volume of distribution of Prazosin. They have instead determined the terminal (non-equilibrium) volume of distribution, $V_{\beta}(47.9 \mathrm{~L})$, most probably using the AUC of the central compartment. Using their assessed pharmacokinetic parameters, we have calculated a $V_{d, 1}$ using Equation (64) of 31.3 L, which is very close to our value. These results are in agreement with the notion that the apparent volume of distribution is always smaller than the $V_{\beta}$ which is calculated during intercompartmental pseudo-equilibrium steady-state conditions [21].

The particular solutions and real-time series equations to drug concentration after multiple intermittent constant rate constant dosing frequency infusions that we have recently developed have allowed us to construct the infusion and elimination phases of plasma or central compartment prazosin concentration as a function of infusion dose number and total time using Equation (76) and Equation (77), respectively, in an open two-compartment model. Similarly, Equations (78)-(79) were used to simulate the peripheral compartment prazosin concentration as a function of total time (Figure 6) [3].

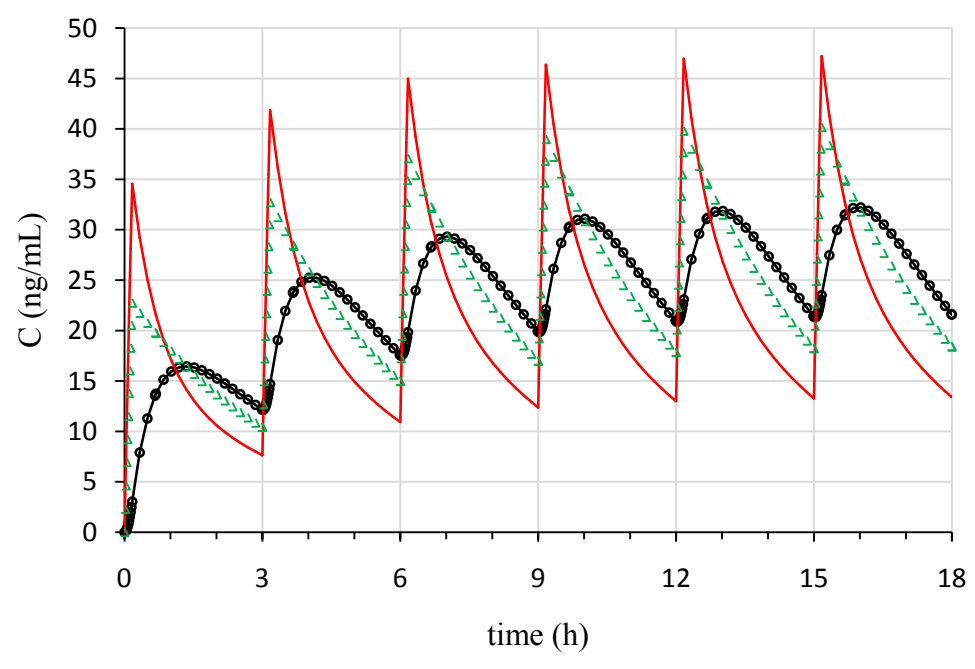

Figure 6. Plasma Prazosin concentration in the central compartment (solid lines), in the peripheral compartment (empty circles joined with solid line) in the two-compartment model and plasma drug concentration in the onecompartment model (empty triangles) after multiple administration of 10minute intermittent infusions with a dosing interval of 3 hours. The ascending part of the drug infusion curves was constructed using the pharmacokinetic parameters assessed from the elimination phase of the drug. 


$$
\begin{aligned}
C_{a, 1, n}(t)= & \sum_{n=1}^{\infty} \frac{\left(k_{21}-\lambda_{1}\right) \cdot C_{1}((n-1) \cdot \tau)+k_{21} \cdot C_{2}((n-1) \cdot \tau)}{\left(\lambda_{2}-\lambda_{1}\right)} \cdot \mathrm{e}^{-\lambda_{1} \cdot(t-(n-1) \cdot \tau)} \\
& +\frac{\left(k_{21}-\lambda_{2}\right) \cdot C_{1}((n-1) \cdot \tau)+k_{21} \cdot C_{2}((n-1) \cdot \tau)}{\left(\lambda_{1}-\lambda_{2}\right)} \cdot \mathrm{e}^{-\lambda_{2} \cdot(t-(n-1) \cdot \tau)} \\
& +\frac{k_{0}}{V_{1}} \cdot\left(\frac{k_{21}}{\lambda_{1} \cdot \lambda_{2}}+\frac{k_{21}-\lambda_{1}}{\lambda_{1} \cdot\left(\lambda_{1}-\lambda_{2}\right)} \cdot \mathrm{e}^{-\lambda_{1} \cdot(t-(n-1) \cdot \tau)}+\frac{k_{21}-\lambda_{2}}{\lambda_{2} \cdot\left(\lambda_{2}-\lambda_{1}\right)} \cdot \mathrm{e}^{-\lambda_{2} \cdot(t-(n-1) \cdot \tau)}\right) \\
C_{e, 1, n}(t)= & \sum_{n=1}^{\infty} \frac{\left(k_{21}-\lambda_{1}\right) \cdot C_{1}((n-1) \cdot \tau+T)+k_{21} \cdot C_{2}((n-1) \cdot \tau+T)}{\left(\lambda_{2}-\lambda_{1}\right)} \cdot \mathrm{e}^{-\lambda_{1} \cdot(t-(n-1) \cdot \tau-T)} \\
& +\frac{\left(k_{21}-\lambda_{2}\right) \cdot C_{1}((n-1) \cdot \tau+T)+k_{21} \cdot C_{2}((n-1) \cdot \tau+T)}{\left(\lambda_{1}-\lambda_{2}\right)} \cdot \mathrm{e}^{-\lambda_{2} \cdot(t-(n-1) \cdot \tau-T)} \\
C_{a, 2, n}(t)= & \sum_{n=1}^{\infty} \frac{\left(\lambda_{2}-k_{21}\right) \cdot C_{2}((n-1) \cdot \tau)+k_{12} \cdot C_{1}((n-1) \cdot \tau)}{\left(\lambda_{2}-\lambda_{1}\right)} \cdot \mathrm{e}^{-\lambda_{1} \cdot(t-(n-1) \cdot \tau)} \\
+ & \frac{\left(\lambda_{1}-k_{21}\right) \cdot C_{2}((n-1) \cdot \tau)+k_{12} \cdot C_{1}((n-1) \cdot \tau)}{\left(\lambda_{1}-\lambda_{2}\right)} \cdot \mathrm{e}^{-\lambda_{2} \cdot(t-(n-1) \cdot \tau)} \\
+ & \frac{k_{0} \cdot k_{12}}{V_{2}} \cdot\left(\frac{1}{\lambda_{1} \cdot \lambda_{2}}+\frac{1}{\lambda_{1} \cdot\left(\lambda_{1}-\lambda_{2}\right)} \cdot \mathrm{e}^{-\lambda_{1} \cdot(t-(n-1) \cdot \tau)}+\frac{1}{\lambda_{2} \cdot\left(\lambda_{2}-\lambda_{1}\right)} \cdot \mathrm{e}^{-\lambda_{2} \cdot(t-(n-1) \cdot \tau)}\right) \\
& +\frac{\left(\lambda_{1}-k_{21}\right) \cdot C_{2}((n-1) \cdot \tau+T)+k_{12} \cdot C_{1}((n-1) \cdot \tau+T)}{\left(\lambda_{1}-\lambda_{2}\right)} \cdot \mathrm{e}^{-\lambda_{2} \cdot(t-(n-1) \cdot \tau-T)} \\
C_{e, 2, n}(t)= & \sum_{n=1}^{\infty} \frac{\left(\lambda_{2}-k_{21}\right) \cdot C_{2}((n-1) \cdot \tau+T)+k_{12} \cdot C_{1}((n-1) \cdot \tau+T)}{\left(\lambda_{2}-\lambda_{1}\right)} \cdot \mathrm{e}^{-\lambda_{1} \cdot(t-(n-1) \cdot \tau-T)}
\end{aligned}
$$

Having assessed the value of the drug apparent volume of distribution, $V_{d, 1}$, from the two-compartment model, we were able to use our recently developed explicit solutions to drug concentration and run comparison simulations after multiple intermittent infusions in the one-compartment model [14]. As shown in Figure 6, the one-compartment model simulations resulted in peak and trough concentrations that are lower and higher than the corresponding peak and trough central compartment concentration. The one-compartment model multiple intermittent infusion simulations were carried out using Equations (80)-(81) for the ascending and descending curves, respectively [14]. The fluctuation factor difference between the two models is not as pronounced as what we have seen with the IV bolus administration. Coincidentally, the 10-minute constant rate of drug infusion that was used in the study is about the same as the distribution half-life $t_{1 / 2, \alpha}$ of the drug. Thus, the plasma drug maximum concentration is much lower than that observed after an IV bolus dose considering that the IV dose $(0.5 \mathrm{mg})$ was only half the dose that was administered by intermittent infusion.

The body exposure to the drug was determined for the one- and the twocompartment model after intermittent intravenous infusions with Equations (82)-(84). In agreement with Gibaldi's work the AUC after IV bolus administration was comparable to that after intermittent infusion (double dose (Table 5) 
[3] [14] [16]. The similarity in the results of the two pharmacokinetic models suggests that Prazosin intermittent infusion dosage calculations can be safely carried out in the clinic with the much simpler one-compartment model equations.

$$
\begin{gathered}
C_{a, n, t}=\frac{k_{0}}{k \cdot V_{d, 1}} \cdot\left(1-\mathrm{e}^{-k \cdot T}\right) \cdot \frac{\left(1-\mathrm{e}^{-k \cdot(n-1) \cdot \tau}\right)}{\left(1-\mathrm{e}^{-k \cdot \tau}\right)} \cdot \mathrm{e}^{-k \cdot(t-(n-2) \cdot \tau-T)} \\
+\frac{k_{0}}{C L} \cdot\left(1-\mathrm{e}^{-k \cdot(t-(n-1) \cdot \tau)}\right) \\
C_{e, n, t}=\frac{k_{0}}{k \cdot V_{d, 1}} \cdot\left(1-\mathrm{e}^{-k \cdot T}\right) \cdot \frac{\left(1-\mathrm{e}^{-n \cdot k \cdot \tau}\right)}{\left(1-\mathrm{e}^{-k \cdot \tau}\right)} \cdot \mathrm{e}^{-k \cdot(t-(n-1) \cdot \tau-T)} \\
\mathrm{AUC}_{\text {one-comp }, n}=T \cdot \frac{k_{0}}{C L}-\frac{k_{0}}{k \cdot C L} \cdot\left(1-\mathrm{e}^{-k \cdot T}\right) \cdot \mathrm{e}^{-k \cdot(n \cdot \tau-T)} \\
\mathrm{AUC} \mathrm{C}_{1, \tau, n}=\frac{\left(k_{21}-\lambda_{1}\right) \cdot C_{e, 1}((n-1) \cdot \tau)+k_{21} \cdot C_{e, 2}((n-1) \cdot \tau)}{\lambda_{1} \cdot\left(\lambda_{2}-\lambda_{1}\right)} \cdot\left(1-\mathrm{e}^{-\lambda_{1} \cdot T}\right) \\
+\frac{\left(k_{21}-\lambda_{2}\right) \cdot C_{e, 1}((n-1) \cdot \tau)+k_{21} \cdot C_{e, 2}((n-1) \cdot \tau)}{\lambda_{2} \cdot\left(\lambda_{1}-\lambda_{2}\right)} \cdot\left(1-\mathrm{e}^{-\lambda_{2} \cdot T}\right) \\
+\frac{k_{0}}{V_{1}} \cdot\left(\frac{k_{21}}{\lambda_{1} \cdot \lambda_{2}} \cdot T+\frac{k_{21}-\lambda_{1}}{\lambda_{1}^{2} \cdot\left(\lambda_{1}-\lambda_{2}\right)} \cdot\left(1-\mathrm{e}^{-\lambda_{1} \cdot T}\right)+\frac{k_{21}-\lambda_{2}}{\lambda_{2}^{2} \cdot\left(\lambda_{2}-\lambda_{1}\right)} \cdot\left(1-\mathrm{e}^{-\lambda_{2} \cdot T}\right)\right) \\
+\frac{\left(k_{21}-\lambda_{1}\right) \cdot C_{a, 1}((n-1) \cdot \tau+T)+k_{21} \cdot C_{a, 2}((n-1) \cdot \tau+T)}{\lambda_{1} \cdot\left(\lambda_{2}-\lambda_{1}\right)} \cdot\left(1-\mathrm{e}^{-\lambda_{1} \cdot(\tau-T)}\right) \\
+\frac{\left(k_{10}+k_{12}-\lambda_{2}\right) \cdot C_{a, 2}((n-1) \cdot \tau+T)+k_{12} \cdot C_{a, 1}((n-1) \cdot \tau+T)}{\lambda_{2} \cdot\left(\lambda_{1}-\lambda_{2}\right)} \cdot\left(1-\mathrm{e}^{-\lambda_{2} \cdot(\tau-T)}\right) \\
+\frac{\left(k_{21}-\lambda_{2}\right) \cdot C_{a, 1}((n-1) \cdot \tau+T)+k_{21} \cdot C_{a, 2}((n-1) \cdot \tau+T)}{\lambda_{2} \cdot\left(\lambda_{1}-\lambda_{2}\right)} \cdot\left(1-\mathrm{e}^{-\lambda_{2} \cdot(\tau-T)}\right) \\
+\frac{\left(k_{10}+k_{12}-\lambda_{2}\right) \cdot C_{e, 2}((n-1) \cdot \tau)+k_{12} \cdot C_{e, 1}((n-1) \cdot \tau)}{\lambda_{2} \cdot\left(\lambda_{1}-\lambda_{2}\right)} \cdot\left(\frac{1}{\lambda_{1} \cdot \lambda_{2}} \cdot T+\frac{\left.\mathrm{e}^{-\lambda_{2} \cdot T}\right)}{\lambda_{1}^{2} \cdot\left(\lambda_{1}-\lambda_{2}\right)} \cdot\left(1-\mathrm{e}^{-\lambda_{1} \cdot T}\right)+\frac{1}{\lambda_{2}^{2} \cdot\left(\lambda_{2}-\lambda_{1}\right)} \cdot\left(1-\mathrm{e}^{-\lambda_{2} \cdot T}\right)\right) \\
\mathrm{AUC}_{2, \tau, n}=\frac{\left(k_{10}+k_{12}-\lambda_{1}\right) \cdot C_{e, 2}((n-1) \cdot \tau)+k_{12} \cdot C_{e, 1}((n-1) \cdot \tau)}{\lambda_{1} \cdot\left(\lambda_{2}-\lambda_{1}\right)} \cdot\left(1-\mathrm{e}^{-\lambda_{1} \cdot T}\right)
\end{gathered}
$$

\subsection{Single $1 \mathrm{mg}$ Oral Prazosin Tablet Administration}

As pointed out in the results section (Figure 3), the simulated rate of absorption was lower than that indicated by the experimental data points. Prazosin is known to be a substrate of efflux transporters but not of influx transporters in the GI [23]. The scarcity of the samples taken during the absorption phase of the drug has made our analysis difficult. Among the twenty or so published clinical 
Table 5. AUC (ng/mL.h) after repetitive $1 \mathrm{mg}$ Prazosin intermittent intravenous infusions at the end of each dosing interval $(\tau=3 \mathrm{~h})$ in the one- and the two-compartment model, as a function of infusion number $(n)$.

\begin{tabular}{cccc}
\hline$n$ & $\mathrm{AUC}_{\text {one_comp }}$ & $\mathrm{AUC}_{1, \tau, n}$ & $\mathrm{AUC}_{2, \tau, n}$ \\
\hline 1 & 46.7 & 46.7 & 48.2 \\
2 & 67.9 & 67.0 & 66.3 \\
3 & 77.2 & 75.9 & 74.2 \\
4 & 81.3 & 79.8 & 77.7 \\
5 & 83.1 & 81.4 & 79.2 \\
6 & 83.9 & 82.2 & 79.8 \\
12 & 84.5 & 82.8 & 80.4 \\
\hline
\end{tabular}

studies after oral administration of Prazosin the most comprehensive one was that of Bateman and coworkers. Still three concentration-time points are not enough to accurately fit the absorption phase into a pharmacokinetic compartment model. An additional problem is that we are using the average results. Although the standard error of those averages appears to be relatively small the standard error is always smaller than the standard deviation by the square root of the total number of samples, that is, by 2.45 for 6 patients. Contrary to the absorption phase, the elimination phase of the drug after oral administration is simulated relatively accurately by both models.

The concentration-time profile after repetitive oral administration of $1 \mathrm{mg}$ Prazosin tablet is simulated in the two-compartment model using Equation (37) and Equation (38), for the central and peripheral plasma drug concentration, respectively (Figure 7). The simulated drug concentration with time in the two compartments after repetitive oral administration of prazosin is very different from the profile in Figure 4 and Figure 6. Both plasma drug concentration and drug concentration in the peripheral compartment are much lower than those achieved after multiple IV boluses or multiple intermittent infusions mainly because of reduced absorption. The regressed bioavailability of the oral administration was only $61.4 \%$ which was very close to the one calculated experimentally by Bateman. Also, due to a lower rate of drug absorption as compared to the rate of intermittent drug infusion $\left(k_{a}=0.5335 \mathrm{~h}^{-1}, t_{1 / 2, \text { absorption }}=1.3 \mathrm{~h}\right)$ and a slightly higher elimination rate constant $\lambda_{2}$, the levels of drug concentration achieved in both compartments was similar.

Momentary distribution equilibrium $\left(t_{e q}\right)$ or $t_{2, n, \max }$ was calculated as a function of time and dose number to be equal to $2.43 \mathrm{~h}, 1.57 \mathrm{~h}, 1.38 \mathrm{~h}$ and $1.37 \mathrm{~h}$ after administration of the $1^{\text {st }}, 3^{\text {rd }}, 6^{\text {th }}$ dose and at steady-state plasma drug concentration, respectively (Figure 7). Simulation of drug concentration with time upon repetitive oral doses in the one-compartment model were carried out with our recently developed Equation (85) [15]. The drug concentration profile in the one-compartment model was very similar to the central compartment concentration but not identical. In particular, the non-steady state central compartment 
peak concentration is slightly lower than the one simulated by the one-compartment model. As steady state conditions are approached with successive oral dosing, central compartment drug concentration reaches higher levels while the fluctuation factor is identical in both models (Figure 7). Steady state concentration in the central compartment and in the one-compartment model were simulated using Equation (39) and Equation (86), respectively.

The drug exposure of the body after repetitive oral administration was assessed by the AUC, and it was much lower than that calculated after multiple IV boluses and multiple intermittent infusions. The AUC per dosing interval was calculated with Equations (45)-(48) for the non-steady state and steady state of central and peripheral compartments, respectively, whereas the AUC in the one-compartment model was calculated using Equations (87)-(88) (Table 6).

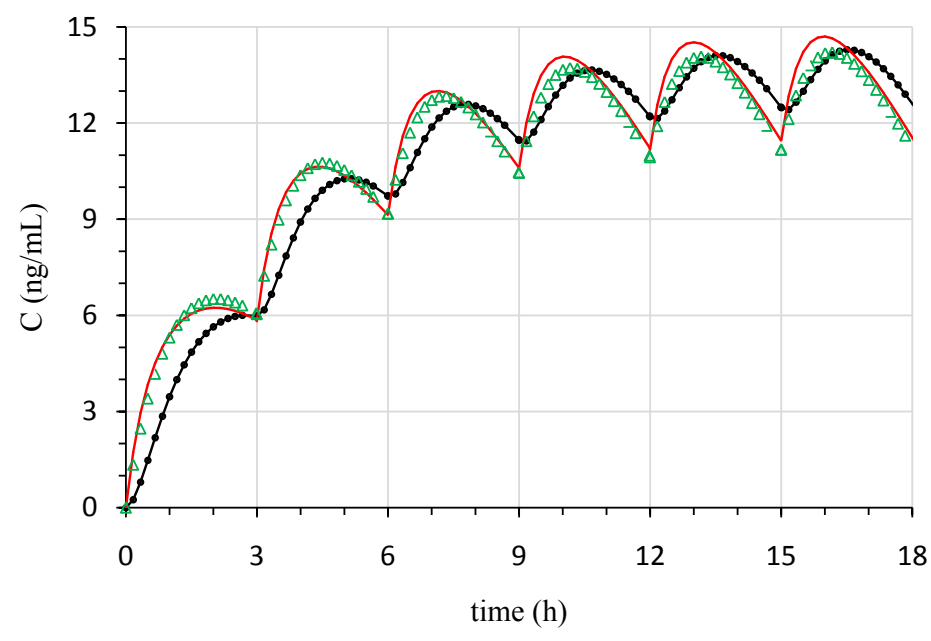

Figure 7. Plasma drug concentration in the central compartment (solid lines), in the peripheral compartment (empty circles joined with solid line) in a two-compartment model and plasma drug concentration in a one-compartment model (empty triangles) after repetitive $1 \mathrm{mg}$ oral tablet administration of Prazosin at a 3-h dosing interval.

Table 6. Calculated non-steady state and steady state AUC $(\mathrm{ng} / \mathrm{mL} \cdot \mathrm{h})$ per dosing interval ( $\tau=3 \mathrm{~h}$ ) after repetitive $1 \mathrm{mg}$ Prazosin oral tablet the one- and two-compartment models, as a function of dose number.

\begin{tabular}{cccc}
\hline$n$ & $\mathrm{AUC}_{\text {one_comp }}$ & $\mathrm{AUC}_{1, \tau}$ & $\mathrm{AUC}_{2, \tau}$ \\
\hline 1 & 15.6 & 15.6 & 7.4 \\
2 & 29.3 & 29.3 & 16.5 \\
3 & 35.5 & 36.1 & 21.4 \\
4 & 38.1 & 39.1 & 23.5 \\
5 & 39.1 & 40.3 & 24.4 \\
6 & 39.4 & 40.7 & 24.7 \\
Steady state & 39.7 & 41.0 & 24.9 \\
\hline
\end{tabular}




$$
\begin{gathered}
C_{n, t}=\frac{F \cdot D \cdot k_{a}}{V_{d} \cdot\left(k_{a}-k\right)} \cdot\left\{\mathrm{e}^{-k \cdot(t-(n-1) \cdot \tau)} \cdot \frac{\left(1-\mathrm{e}^{-k \cdot n \cdot \tau}\right)}{\left(1-\mathrm{e}^{-k \cdot \tau}\right)}-\mathrm{e}^{-k_{a} \cdot(t-(n-1) \cdot \tau)} \cdot \frac{\left(1-\mathrm{e}^{-k_{a} \cdot n \cdot \tau}\right)}{\left(1-\mathrm{e}^{-k_{a} \cdot \tau}\right)}\right\} \\
C_{s s, n, t}=\frac{F \cdot D \cdot k_{a}}{V_{d} \cdot\left(k_{a}-k\right)} \cdot\left\{\frac{\mathrm{e}^{-k \cdot(t-(n-1) \cdot \tau)}}{\left(1-\mathrm{e}^{-k \cdot \tau}\right)}-\frac{\mathrm{e}^{-k_{a} \cdot(t-(n-1) \cdot \tau)}}{\left(1-\mathrm{e}^{-k_{a} \cdot \tau}\right)}\right\} \\
\mathrm{AUC}_{\text {one-comp }, \tau, n}=\frac{F \cdot D \cdot k_{a}}{V_{d} \cdot\left(k_{a}-k\right)} \cdot\left[\frac{\left(1-\mathrm{e}^{-k \cdot n \cdot \tau}\right)}{k}-\frac{\left(1-\mathrm{e}^{-k_{a} \cdot n \cdot \tau}\right)}{k_{a}}\right] \\
\mathrm{AUC}_{\text {one-comp }, \tau, s s}=\frac{F \cdot D \cdot k_{a}}{V_{d} \cdot\left(k_{a}-k\right)} \cdot\left[\frac{1}{k}-\frac{1}{k_{a}}\right]
\end{gathered}
$$

\section{Conclusions}

The pharmacokinetic parameters of the drug Prazosin were determined from published clinical data after intravenous bolus injection, intermittent infusion and oral administration using a two-compartment model [5] [6]. It is the first time that steady-state drug concentration equations were derived for all routes of administration in a two-compartment mammillary pharmacokinetic model. With regard to the IV bolus study, our results were similar to those published by Grahnen et al., and we have obtained many additional pharmacokinetic parameters. In particular, we have determined the peak time and peak peripheral drug concentration, the AUC and most importantly the apparent volume of distribution of Prazosin from an open two-compartment model. As far as the single intermittent intravenous infusion study is concerned, the data were fitted with nonlinear regression analysis using the particular solutions of the elimination phase of the drug. The extracted pharmacokinetic parameters were subsequently used to construct the ascending phase during the infusion time period of Prazo$\sin$ [3]. As for the clinical study of the oral route of administration, several time-dependent pharmacokinetic parameters such as peak time and peak concentration in both the central and peripheral compartments were determined using real-time analytical solutions to drug concentration as a function of oral dose number [15].

The current explicit series solutions in an open two-compartment pharmacokinetic model described herein have allowed non-steady state and steady state simulations of repetitive drug doses at constant dosing frequency for all three routes of administration. In an original way, the value of the apparent volume of distribution of Prazosin was determined from a two-compartment pharmacokinetic model. Its significance is two-fold: first, with a value being close to that of total body water it is suggested that Prazosin is distributed both extracellularly and intracellularly, in all tissues, and second, the apparent volume of distribution allowed simulations in the one-compartment model. It was shown that dosage calculations of Prazosin intermittent infusions can be safely managed using the much simpler one-compartment model. 


\section{Conflicts of Interest}

The authors declare no conflicts of interest regarding the publication of this paper.

\section{References}

[1] Savva, M. (2021) On the Origin of the Apparent Volume of Distribution and Its Significance in Solvent Extraction Methods. Journal of Organic Chemistry and Chemical Sciences, 1, 101. https://doi.org/10.17303/jocs.2021.1.101

[2] Savva, M. (2022) On the Origin of the Apparent Volume of Distribution and Its Significance in Pharmacokinetics. Journal of Biosciences and Medicines. (In Press)

[3] Savva, M. (2021) Real-Time Analytical Solutions as Series Formulas and Heaviside off/on Switch Functions for Multiple Intermittent Intravenous Infusions. Journal of Biosciences and Medicines. (In Press)

[4] Langer, S.Z., Cavero, I. and Massingham, R. (1980) Recent Developments in Noradrenergic Neurotransmission and Its Relevance to the Mechanism of Action of Certain Antihypertensive Agents. Hypertension, 2, 372-382.

https://doi.org/10.1161/01.HYP.2.4.372

[5] Bateman, D.N., Hobbs, D.C., Twomey, T.M., Stevens, E.A. and Rawlins, M.D. (1979) Prazosin, Pharmacokinetics and Concentration Effect. European Journal of Clinical Pharmacology, 16, 177-181. https://doi.org/10.1007/BF00562058

[6] Grahnen, A., Seideman, P., Lindstrom, B., Haglund, K. and von Bahr, C. (1981) Prazosin Kinetics in Hypertension. Clinical Pharmacology \& Therapeutics, 30, 439-446. https://doi.org/10.1038/clpt.1981.186

[7] Hobbs, D.C., Twomey, T.M. and Palmer, R.F. (1978) Pharmacokinetics of Prazosin in Man. The Journal of Clinical Pharmacology, 18, 402-406. https://doi.org/10.1002/j.1552-4604.1978.tb02456.x

[8] Baughman, R.A., Arnold, S., Benet, L.Z., Lin, E.T., Chatterjee, K. and Williams, R.L. (1980) Altered Prazosin Pharmacokinetics in Congestive Heart Failure. European Journal of Clinical Pharmacology, 17, 425-428. https://doi.org/10.1007/BF00570159

[9] Chau, N.P., Flouvat, B.L., Roux, E.L. and Safar, M.E. (1980) Prazosin Kinetics in Essential Hypertension. Clinical Pharmacology \& Therapeutics, 28, 6-11. https://doi.org/10.1038/clpt.1980.123

[10] Dynon, M.K., Jarrott, B., Drummer, O. and Louis, W.J. (1980) Pharmacokinetics of Prazosin in Normotensive Subjects after Low Oral Doses. Clinical Pharmacology \& Therapeutics, 5, 583-590. https://doi.org/10.2165/00003088-198005060-00007

[11] Guelen, P.J.M., Janssen, T.J., Lam, M.H., Vree, T.B. and Exler, P.S. (1990) Comparative Bioavailability of Two Brands of Prazosin-Containing Tablets in Healthy Volunteers. Pharmaceutisch Weekblad Scientific, 12, 184-187. https://doi.org/10.1007/BF01980043

[12] Dynon, M.K., Jarrott, B. and Louis, W.J. (1983) Tissue Distribution and Hypotensive Effect of Prazosin in the Conscious Rat. Journal of Cardiovascular Pharmacology and Therapeutics, 5, 235-239. https://doi.org/10.1097/00005344-198303000-00012

[13] Graham, R.M., Thornell, J.R., Gain, J.M., Bagnoli, C., Oates, H.F. and Stokes, G.S. (1976) Prazosin: The First Dose Phenomenon. British Medical Journal, 2, 1293-1294. https://doi.org/10.1136/bmj.2.6047.1293

[14] Savva, M. (2021) A Mathematical Treatment of Multiple Intermittent Intravenous 
Infusions in a One-Compartment Model. Computer Methods and Programs in Biomedicine, 205, Article ID: 106103. https://doi.org/10.1016/j.cmpb.2021.106103

[15] Savva, M. (2021) A Method to Custom-Build Equations of Drug Concentration as a Function of Real Time in the One-Compartment Open Model and Their Application in Multiple Intravenous Bolus and Multiple Oral Doses. Pharmaceutical and Biosciences Journal, 9, 12-29.

https://pharmabiosciencejournal.com/index.php/pbj/article/view/2284

[16] Riggs, D.S. (1963) Transfer of Substances between Biological Compartments. General Kinetics. In: The Mathematical Approach to Physiological Problems, Williams and Wilkins, Baltimore, 193-217.

[17] Grimm Jr., R.H. (1989) $\alpha_{1}$-Antagonists in the Treatment of Hypertension. Hypertension, 13, I131-I136. https://doi.org/10.1161/01.HYP.13.5_Suppl.I131

[18] Akinaga, J., Garcia-Sainz, J.A. and Pupo, A.S. (2019) Updates in the Function and Regulation of $\alpha_{1}$-Adrenoceptors. British Journal of Pharmacology, 176, 2343-2357. https://doi.org/10.1111/bph.14617

[19] Lameire, N. and Gordts, J. (1986) A Pharmacokinetic Study of Prazosin with Varying Degrees of Chronic Renal Failure. European Journal of Clinical Pharmacology, 31, 333-327. https://doi.org/10.1007/BF00981133

[20] Horowitz, J.D., Dynon, M.K., Jarrott, B., Brennan, J.B., Oliver, L.E., Goble, A.J. and Louis, W.J. (1984) Haemodynamic Effects of a Single Low Dose of Prazosin with Chronic Congestive Cardiac Failure Correlations with Pharmacokinetics. Clinical and Experimental Pharmacology and Physiology, 11, 7-15.

https://doi.org/10.1111/j.1440-1681.1984.tb00234.x

[21] Gibaldi, M. (1969) Effect of Mode of Administration on Drug Distribution in a Two-Compartment Open System. Journal of Pharmaceutical Sciences, 58, 327-331. https://doi.org/10.1002/jps.2600580308

[22] Riegelman, S., Loo, J. and Rowland, M. (1968) Concept of a Volume of Distribution and Possible Errors in Evaluation of This Parameter. Journal of Pharmaceutical Sciences, 57, 128-133. https://doi.org/10.1002/jps.2600570125

[23] Speer, J.E., Gunasekara, D.B., Wang, Y., Fallon, J.K., Attayek, P.J., Smith, P.C., Sims, C.E. and Allbritton, N.L. (2019) Molecular Transport through Primary Human Small Intestinal Monolayers by Culture on a Collagen Scaffold with a Gradient of Chemical Cross-Linking. Journal of Biological Engineering, 13, 36.

https://doi.org/10.1186/s13036-019-0165-4 


\section{Appendix}

Time to reach peak concentration in the peripheral compartment as a function of IV bolus dose number $(n)$.

$$
\begin{aligned}
& k_{12} \cdot x_{1}-k_{21} \cdot x_{2}=0 \\
& k_{12} \cdot D \cdot\left\{A_{1} \cdot\left(\frac{\left(1-\mathrm{e}^{-\lambda_{1} \cdot n \cdot \tau}\right)}{\left(1-\mathrm{e}^{-\lambda_{1} \cdot \tau}\right)} \cdot \mathrm{e}^{-\lambda_{1} \cdot(t-(n-1) \cdot \tau)}\right)+B_{1} \cdot\left(\frac{\left(1-\mathrm{e}^{-\lambda_{2} \cdot n \cdot \tau}\right)}{\left(1-\mathrm{e}^{-\lambda_{2} \cdot \tau}\right)} \cdot \mathrm{e}^{-\lambda_{2} \cdot(t-(n-1) \cdot \tau)}\right)\right\} \\
& =k_{21} \cdot D \cdot k_{12} \cdot\left\{A_{2} \cdot \frac{\left(1-\mathrm{e}^{-\lambda_{1} \cdot n \cdot \tau}\right)}{\left(1-\mathrm{e}^{-\lambda_{1} \cdot \tau}\right)} \cdot \mathrm{e}^{-\lambda_{1} \cdot(t-(n-1) \cdot \tau)}+B_{2} \cdot \frac{\left(1-\mathrm{e}^{-\lambda_{2} \cdot n \cdot \tau}\right)}{\left(1-\mathrm{e}^{-\lambda_{2} \cdot \tau}\right)} \cdot \mathrm{e}^{-\lambda_{2} \cdot(t-(n-1) \cdot \tau)}\right\} \\
& {\left[A_{1} \cdot \frac{\left(1-\mathrm{e}^{-\lambda_{1} \cdot n \cdot \tau}\right)}{\left(1-\mathrm{e}^{-\lambda_{1} \cdot \tau}\right)}-k_{21} \cdot A_{2} \cdot \frac{\left(1-\mathrm{e}^{-\lambda_{1} \cdot n \cdot \tau}\right)}{\left(1-\mathrm{e}^{-\lambda_{1} \cdot \tau}\right)}\right] \cdot \mathrm{e}^{-\lambda_{1} \cdot(t-(n-1) \cdot \tau)}} \\
& =\left[k_{21} \cdot B_{2} \cdot \frac{\left(1-\mathrm{e}^{-\lambda_{2} \cdot n \cdot \tau}\right)}{\left(1-\mathrm{e}^{-\lambda_{2} \cdot \tau}\right)}-B_{1} \cdot \frac{\left(1-\mathrm{e}^{-\lambda_{2} \cdot n \cdot \tau}\right)}{\left(1-\mathrm{e}^{-\lambda_{2} \cdot \tau}\right)}\right] \cdot \mathrm{e}^{-\lambda_{2} \cdot(t-(n-1) \cdot \tau)} \\
& {\left[A_{1}-k_{21} \cdot A_{2}\right] \cdot \frac{\left(1-\mathrm{e}^{-\lambda_{1} \cdot n \cdot \tau}\right)}{\left(1-\mathrm{e}^{-\lambda_{1} \cdot \tau}\right)} \cdot \mathrm{e}^{-\lambda_{1} \cdot(t-(n-1) \cdot \tau)}} \\
& =\left[k_{21} \cdot B_{2}-B_{1}\right] \cdot \frac{\left(1-\mathrm{e}^{-\lambda_{2} \cdot n \cdot \tau}\right)}{\left(1-\mathrm{e}^{-\lambda_{2} \cdot \tau}\right)} \cdot \mathrm{e}^{-\lambda_{2} \cdot(t-(n-1) \cdot \tau)} \\
& {\left[\frac{\lambda_{1}-k_{21}}{\left(\lambda_{1}-\lambda_{2}\right)}-\frac{k_{21}}{\left(\lambda_{2}-\lambda_{1}\right)}\right] \cdot \frac{\left(1-\mathrm{e}^{-\lambda_{1} \cdot n \cdot \tau}\right)}{\left(1-\mathrm{e}^{-\lambda_{1} \cdot \tau}\right)} \cdot \mathrm{e}^{-\lambda_{1} \cdot(t-(n-1) \cdot \tau)}} \\
& =\left[\frac{k_{21}}{\left(\lambda_{1}-\lambda_{2}\right)}-\frac{k_{21}-\lambda_{2}}{\left(\lambda_{1}-\lambda_{2}\right)}\right] \cdot \frac{\left(1-\mathrm{e}^{-\lambda_{2} \cdot n \cdot \tau}\right)}{\left(1-\mathrm{e}^{-\lambda_{2} \cdot \tau}\right)} \cdot \mathrm{e}^{-\lambda_{2} \cdot(t-(n-1) \cdot \tau)} \\
& \frac{\lambda_{1} \cdot\left(1-\mathrm{e}^{-\lambda_{1} \cdot n \cdot \tau}\right)}{\left(\lambda_{1}-\lambda_{2}\right) \cdot\left(1-\mathrm{e}^{-\lambda_{1} \cdot \tau}\right)} \cdot \mathrm{e}^{-\lambda_{1} \cdot(t-(n-1) \cdot \tau)}=\frac{\lambda_{2} \cdot\left(1-\mathrm{e}^{-\lambda_{2} \cdot n \cdot \tau}\right)}{\left(\lambda_{1}-\lambda_{2}\right) \cdot\left(1-\mathrm{e}^{-\lambda_{2} \cdot \tau}\right)} \cdot \mathrm{e}^{-\lambda_{2} \cdot(t-(n-1) \cdot \tau)} \\
& \mathrm{e}^{\left(\lambda_{2}-\lambda_{1}\right) \cdot(t-(n-1) \cdot \tau)}=\frac{\lambda_{2}}{\lambda_{1}} \cdot \frac{\left(1-\mathrm{e}^{-\lambda_{2} \cdot n \cdot \tau}\right)}{\left(1-\mathrm{e}^{-\lambda_{2} \cdot \tau}\right)} \cdot \frac{\left(1-\mathrm{e}^{-\lambda_{1} \cdot \tau}\right)}{\left(1-\mathrm{e}^{-\lambda_{1} \cdot n \cdot \tau}\right)} \\
& t_{2, n, \max }=(n-1) \cdot \tau+\frac{\ln \left(\frac{\lambda_{2} \cdot\left(1-\mathrm{e}^{-\lambda_{2} \cdot n \cdot \tau}\right) \cdot\left(1-\mathrm{e}^{-\lambda_{1} \cdot \tau}\right)}{\lambda_{1} \cdot\left(1-\mathrm{e}^{-\lambda_{2} \cdot \tau}\right) \cdot\left(1-\mathrm{e}^{-\lambda_{1} \cdot n \cdot \tau}\right)}\right)}{\left(\lambda_{2}-\lambda_{1}\right)}
\end{aligned}
$$

Multiple Oral administration in a two-compartment mammillary model Differential equations for inputs and outputs.

Input: First-order drug absorption into the central compartment.

Output: First-order drug elimination from the central compartment.

$$
\begin{gathered}
\dot{x}_{1}=-\left(k_{10}+k_{12}\right) \cdot x_{1}+k_{21} \cdot x_{2}+k_{a} \cdot x_{3} ; \quad x_{1}(0)=0 \\
\dot{x}_{2}=k_{12} \cdot x_{1}-k_{21} \cdot x_{2} ; \quad x_{2}(0)=0
\end{gathered}
$$




$$
\dot{x}_{3}=-k_{a} \cdot x_{3} ; \quad x_{3}(0)=D
$$

where $D$ is the drug dose and $k_{a}$ is the first-order absorption rate constant.

Analytical solutions and sequence terms for multiple oral doses

Equations (31)-(33) can be written in matrix form $\vec{x}^{\prime}=M \cdot \vec{x}$ :

$$
\vec{x}^{\prime}=\left(\begin{array}{ccc}
-\left(k_{10}+k_{12}\right) & k_{21} & k_{a} \\
k_{12} & -k_{21} & 0 \\
0 & 0 & -k_{a}
\end{array}\right) \cdot \vec{x} ; \vec{x}(0)=\left(\begin{array}{l}
0 \\
0 \\
D
\end{array}\right)
$$

Applying the Laplace transform,

$$
\left(\begin{array}{ccc}
\left(s+k_{10}+k_{12}\right) & -k_{21} & -k_{a} \\
-k_{12} & \left(s+k_{21}\right) & 0 \\
0 & 0 & \left(s+k_{a}\right)
\end{array}\right) \cdot \vec{X}(s)=\left(\begin{array}{c}
0 \\
0 \\
D
\end{array}\right)
$$

Using the Cramer's rule,

$$
\begin{gathered}
X_{1}(s)=\frac{\operatorname{det}\left(M_{1}\right)}{\operatorname{det}(M)} ; X_{2}(s)=\frac{\operatorname{det}\left(M_{2}\right)}{\operatorname{det}(M)} ; X_{3}(s)=\frac{\operatorname{det}\left(M_{3}\right)}{\operatorname{det}(M)} \\
M=\left(\begin{array}{ccc}
\left(s+k_{10}+k_{12}\right) & -k_{21} & -k_{a} \\
-k_{12} & \left(s+k_{21}\right) & 0 \\
0 & 0 & \left(s+k_{a}\right)
\end{array}\right) \\
M_{1}=\left(\begin{array}{ccc}
0 & -k_{21} & -k_{a} \\
0 & \left(s+k_{21}\right) & 0 \\
D & 0 & \left(s+k_{a}\right)
\end{array}\right) \\
M_{2}=\left(\begin{array}{ccc}
\left(s+k_{10}+k_{12}\right) & 0 & -k_{a} \\
-k_{12} & 0 & 0 \\
0 & D & \left(s+k_{a}\right)
\end{array}\right) \\
\operatorname{det}(M)=\left(s+k_{a}\right) \cdot\left(s^{2}+\left(k_{10}+k_{12}+k_{21}\right) \cdot s+k_{10} \cdot k_{12}\right)
\end{gathered}
$$

Let $\lambda_{1}+\lambda_{2}=k_{10}+k_{12}+k_{21}$ and $\lambda_{1} \cdot \lambda_{2}=k_{10} \cdot k_{12}$

$$
\begin{gathered}
\operatorname{det}(M)=\left(s+k_{a}\right) \cdot\left(s+\lambda_{1}\right) \cdot\left(s+\lambda_{2}\right) \\
\operatorname{det}\left(M_{1}\right)=D \cdot k_{a} \cdot\left(s+k_{21}\right) \\
\operatorname{det}\left(M_{2}\right)=D \cdot k_{a} \cdot k_{12} \\
\operatorname{det}\left(M_{3}\right)=\left(s+k_{10}+k_{12}\right) \\
X_{1}(s)=\frac{D \cdot k_{a} \cdot\left(s+k_{21}\right)}{\left(s+k_{a}\right) \cdot\left(s+\lambda_{1}\right) \cdot\left(s+\lambda_{2}\right)} ; \quad X_{2}(s)=\frac{D \cdot k_{a} \cdot k_{12}}{\left(s+k_{a}\right) \cdot\left(s+\lambda_{1}\right) \cdot\left(s+\lambda_{2}\right)} ; \\
X_{3}(s)=\frac{\left(s+k_{10}+k_{12}\right)}{\left(s+k_{a}\right) \cdot\left(s+\lambda_{1}\right) \cdot\left(s+\lambda_{2}\right)}
\end{gathered}
$$


Using partial fraction decomposition,

$$
\begin{aligned}
& X_{1}(s)= D \cdot k_{a} \cdot\left[\frac{\left(k_{21}-\lambda_{1}\right)}{\left(s+\lambda_{1}\right) \cdot\left(k_{a}-\lambda_{1}\right) \cdot\left(\lambda_{2}-\lambda_{1}\right)}\right. \\
&\left.+\frac{\left(k_{21}-\lambda_{2}\right)}{\left(s+\lambda_{2}\right) \cdot\left(k_{a}-\lambda_{2}\right) \cdot\left(\lambda_{1}-\lambda_{2}\right)}+\frac{\left(k_{21}-k_{a}\right)}{\left(s+k_{a}\right) \cdot\left(k_{a}-\lambda_{1}\right) \cdot\left(k_{a}-\lambda_{2}\right)}\right] \\
& X_{2}(s)= D \cdot k_{a} \cdot k_{12} \cdot\left[\frac{1}{\left(s+\lambda_{1}\right) \cdot\left(k_{a}-\lambda_{1}\right) \cdot\left(\lambda_{2}-\lambda_{1}\right)}\right. \\
&\left.+\frac{1}{\left(s+\lambda_{2}\right) \cdot\left(k_{a}-\lambda_{2}\right) \cdot\left(\lambda_{1}-\lambda_{2}\right)}+\frac{1}{\left(s+k_{a}\right) \cdot\left(k_{a}-\lambda_{1}\right) \cdot\left(k_{a}-\lambda_{2}\right)}\right] \\
& X_{3}(s)=\frac{D}{\left(s+k_{a}\right)}
\end{aligned}
$$

Taking the inverse transforms,

$$
\begin{gathered}
x_{1}(t)=D \cdot k_{a} \cdot\left(A_{3} \cdot \mathrm{e}^{-\lambda_{1} \cdot t}+B_{3} \cdot \mathrm{e}^{-\lambda_{2} \cdot t}+E_{3} \cdot \mathrm{e}^{-k_{a} \cdot t}\right) \\
A_{3}=\frac{\left(k_{21}-\lambda_{1}\right)}{\left(k_{a}-\lambda_{1}\right) \cdot\left(\lambda_{2}-\lambda_{1}\right)} ; \quad B_{3}=\frac{\left(k_{21}-\lambda_{2}\right)}{\left(k_{a}-\lambda_{2}\right) \cdot\left(\lambda_{1}-\lambda_{2}\right)} ; \quad E_{3}=\frac{\left(k_{21}-k_{a}\right)}{\left(k_{a}-\lambda_{1}\right) \cdot\left(k_{a}-\lambda_{2}\right)} \\
C_{1}(t)=\frac{x_{1}(t)}{V_{1}} \\
x_{2}(t)=D \cdot k_{a} \cdot k_{12} \cdot\left(A_{4} \cdot \mathrm{e}^{-\lambda_{1} \cdot t}+B_{4} \cdot \mathrm{e}^{-\lambda_{2} \cdot t}+E_{4} \cdot \mathrm{e}^{-k_{a} \cdot t}\right) \\
\frac{1}{\left(k_{a}-\lambda_{1}\right) \cdot\left(\lambda_{2}-\lambda_{1}\right)} ; \quad B_{4}=\frac{1}{\left(k_{a}-\lambda_{2}\right) \cdot\left(\lambda_{1}-\lambda_{2}\right)} ; E_{4}=\frac{1}{\left(k_{a}-\lambda_{1}\right) \cdot\left(k_{a}-\lambda_{2}\right)} \\
C_{2}(t)=\frac{x_{2}(t)}{V_{2}} \\
x_{3}(t)=D \cdot \mathrm{e}^{-k_{a} \cdot t} \\
C_{3}(t)=\frac{x_{3}(t)}{V_{3}}
\end{gathered}
$$

Terms of the mathematical sequence for multiple doses administered with a dosing interval $\tau$.

$$
\begin{aligned}
\left\{C_{1, n}\right\}=\{ & \frac{D \cdot k_{a}}{V_{1}} \cdot\left(A_{3} \cdot \mathrm{e}^{-\lambda_{1} \cdot t}+B_{3} \cdot \mathrm{e}^{-\lambda_{2} \cdot t}+E_{3} \cdot \mathrm{e}^{-k_{a} \cdot t}\right), \\
& \frac{D \cdot k_{a}}{V_{1}} \cdot\left(A_{3} \cdot \mathrm{e}^{-\lambda_{1} \cdot t}+B_{3} \cdot \mathrm{e}^{-\lambda_{2} \cdot t}+E_{3} \cdot \mathrm{e}^{-k_{a} \cdot t}\right) \\
& \left.+\frac{D \cdot k_{a}}{V_{1}} \cdot\left(A_{3} \cdot \mathrm{e}^{-\lambda_{1} \cdot(t-\tau)}+B_{3} \cdot \mathrm{e}^{-\lambda_{2} \cdot(t-\tau)}+E_{3} \cdot \mathrm{e}^{-k_{a} \cdot(t-\tau)}\right), \cdots\right\}
\end{aligned}
$$

Pattern of Sequence

$$
\left\{C_{1, n}\right\}=\left\{\frac{F \cdot D \cdot k_{a}}{V_{1}} \cdot\left(A_{3} \cdot \mathrm{e}^{-\lambda_{1} \cdot(t-(n-1) \cdot \tau)}+B_{3} \cdot \mathrm{e}^{-\lambda_{2} \cdot(t-(n-1) \cdot \tau)}+E_{3} \cdot \mathrm{e}^{-k_{a} \cdot(t-(n-1) \cdot \tau)}\right)\right\}
$$


where, $t$ is the total time.

Partial sums and formula of the mathematical Series for repetitive oral doses

$$
C_{1, n}=\sum_{n=1}^{\infty} \frac{D \cdot k_{a}}{V_{1}} \cdot\left(A_{3} \cdot \mathrm{e}^{-\lambda_{1} \cdot(t-(n-1) \cdot \tau)}+B_{3} \cdot \mathrm{e}^{-\lambda_{2} \cdot(t-(n-1) \cdot \tau)}+E_{3} \cdot \mathrm{e}^{-k_{a} \cdot(t-(n-1) \cdot \tau)}\right)
$$

Dividing and multiplying corresponding terms by $\left(1-\mathrm{e}^{-\lambda_{1} \cdot \tau}\right),\left(1-\mathrm{e}^{-\lambda_{2} \cdot \tau}\right)$ and by $\left(1-\mathrm{e}^{-k_{a} \cdot \tau}\right)$, yields the partial sums of the series shown below:

$$
\begin{aligned}
& C_{1, n}=\sum_{n=1}^{\infty} \frac{D \cdot k_{a}}{V_{1}} \cdot\left(A_{3} \cdot \mathrm{e}^{-\lambda_{1} \cdot(t-(n-1) \cdot \tau)}+B_{3} \cdot \mathrm{e}^{-\lambda_{2} \cdot(t-(n-1) \cdot \tau)}+E_{3} \cdot \mathrm{e}^{-k_{a} \cdot(t-(n-1) \cdot \tau)}\right) \\
& =\frac{D \cdot k_{a}}{V_{1}} \cdot\left\{A_{3} \cdot\left(\frac{\mathrm{e}^{-\lambda_{1} \cdot t}+\mathrm{e}^{-\lambda_{1} \cdot(t-\tau)}+\mathrm{e}^{-\lambda_{1} \cdot(t-2 \cdot \tau)}+\cdots+\mathrm{e}^{-\lambda_{1} \cdot(t-(n-1) \cdot \tau)}-\mathrm{e}^{-\lambda_{1} \cdot(t+\tau)}-\mathrm{e}^{-\lambda_{1} \cdot t}-\mathrm{e}^{-\lambda_{1} \cdot(t-\tau)}-\mathrm{e}^{-\lambda_{1} \cdot(t-2 \cdot \tau)}-\cdots-\mathrm{e}^{-\lambda_{1} \cdot(t-(n-2) \cdot \tau)}}{\left(1-\mathrm{e}^{-\lambda_{1} \cdot \tau}\right)}\right)\right. \\
& +B_{3} \cdot\left(\frac{\mathrm{e}^{-\lambda_{2} \cdot t}+\mathrm{e}^{-\lambda_{2} \cdot(t-\tau)}+\cdots+\mathrm{e}^{-\lambda_{2} \cdot(t-(n-1) \cdot \tau)}-\mathrm{e}^{-\lambda_{2} \cdot(t+\tau)}-\mathrm{e}^{-\lambda_{2} \cdot t}-\mathrm{e}^{-\lambda_{2} \cdot(t-\tau)}-\cdots-\mathrm{e}^{-\lambda_{2} \cdot(t-(n-2) \cdot \tau)}}{\left(1-\mathrm{e}^{-\lambda_{2} \cdot \tau}\right)}\right) \\
& \left.+E_{3} \cdot\left(\frac{\mathrm{e}^{-k_{a} \cdot t}+\mathrm{e}^{-k_{a} \cdot(t-\tau)}+\cdots+\mathrm{e}^{-k_{a} \cdot(t-(n-1) \cdot \tau)}-e^{-k_{a} \cdot(t+\tau)}-\mathrm{e}^{-k_{a} \cdot t}-\mathrm{e}^{-k_{a} \cdot(t-\tau)}-\cdots-\mathrm{e}^{-k_{a} \cdot(t-(n-2) \cdot \tau)}}{\left(1-\mathrm{e}^{-k_{a} \cdot \tau}\right)}\right)\right\} \\
& =\frac{D \cdot k_{a}}{V_{1}} \cdot\left\{A_{3} \cdot\left(\frac{\mathrm{e}^{-\lambda_{1} \cdot(t-(n-1) \cdot \tau)}-\mathrm{e}^{-\lambda_{1} \cdot(t+\tau)}}{\left(1-\mathrm{e}^{-\lambda_{1} \cdot \tau}\right)}\right)+B_{3} \cdot\left(\frac{\mathrm{e}^{-\lambda_{2} \cdot(t-(n-1) \cdot \tau)}-\mathrm{e}^{-\lambda_{2} \cdot(t+\tau)}}{\left(1-\mathrm{e}^{-\lambda_{2} \cdot \tau}\right)}\right)+E_{3} \cdot\left(\frac{\mathrm{e}^{-k_{a} \cdot(t-(n-1) \cdot \tau)}-\mathrm{e}^{-k_{a} \cdot(t+\tau)}}{\left(1-\mathrm{e}^{-k_{a} \cdot \tau}\right)}\right)\right\} \\
& C_{1, n, t}=\frac{D \cdot k_{a}}{V_{1}} \cdot\left\{A_{3} \cdot \frac{\left(1-\mathrm{e}^{-\lambda_{1} \cdot n \cdot \tau}\right)}{\left(1-\mathrm{e}^{-\lambda_{1} \cdot \tau}\right)} \cdot \mathrm{e}^{-\lambda_{1} \cdot(t-(n-1) \cdot \tau)}\right. \\
& \left.+B_{3} \cdot \frac{\left(1-\mathrm{e}^{-\lambda_{2} \cdot n \cdot \tau}\right)}{\left(1-\mathrm{e}^{-\lambda_{2} \cdot \tau}\right)} \cdot \mathrm{e}^{-\lambda_{2} \cdot(t-(n-1) \cdot \tau)}+E_{3} \cdot \frac{\left(1-\mathrm{e}^{-k_{a} \cdot n \cdot \tau}\right)}{\left(1-\mathrm{e}^{-k_{a} \cdot \tau}\right)} \cdot \mathrm{e}^{-k_{a} \cdot(t-(n-1) \cdot \tau)}\right\}
\end{aligned}
$$

Similarly,

$$
\begin{gathered}
C_{2, n, t}=\frac{D \cdot k_{a} \cdot k_{12}}{V_{2}} \cdot\left\{A_{4} \cdot \frac{\left(1-\mathrm{e}^{-\lambda_{1} \cdot n \cdot \tau}\right)}{\left(1-\mathrm{e}^{-\lambda_{1} \cdot \tau}\right)} \cdot \mathrm{e}^{-\lambda_{1} \cdot(t-(n-1) \cdot \tau)}\right. \\
\left.+B_{4} \cdot \frac{\left(1-\mathrm{e}^{-\lambda_{2} \cdot n \cdot \tau}\right)}{\left(1-\mathrm{e}^{-\lambda_{2} \cdot \tau}\right)} \cdot \mathrm{e}^{-\lambda_{2} \cdot(t-(n-1) \cdot \tau)}+E_{4} \cdot \frac{\left(1-\mathrm{e}^{-k_{a} \cdot n \cdot \tau}\right)}{\left(1-\mathrm{e}^{-k_{a} \cdot \tau}\right)} \cdot \mathrm{e}^{-k_{a} \cdot(t-(n-1) \cdot \tau)}\right\} \quad \begin{array}{l}
n, t \in[0, \infty) \\
C_{3, n, t}=\frac{D}{V_{3}} \cdot \frac{\left(1-\mathrm{e}^{-k_{a} \cdot n \cdot \tau}\right)}{\left(1-\mathrm{e}^{-k_{a} \cdot \tau}\right)} \cdot \mathrm{e}^{-k_{a} \cdot(t-(n-1) \cdot \tau)} \quad n, t \in[0, \infty)
\end{array}
\end{gathered}
$$

\section{AUC formulas}

$$
\begin{aligned}
\mathrm{AUC}_{1, \max , n}= & \int_{(n-1) \cdot \tau}^{n \cdot \tau} \frac{D \cdot k_{a}}{V_{1}} \cdot\left\{A_{3} \cdot \frac{\left(1-\mathrm{e}^{-\lambda_{1} \cdot n \cdot \tau}\right)}{\left(1-\mathrm{e}^{-\lambda_{1} \cdot \tau}\right)} \cdot \mathrm{e}^{-\lambda_{1} \cdot(t-(n-1) \cdot \tau)}\right. \\
& \left.+B_{3} \cdot \frac{\left(1-\mathrm{e}^{-\lambda_{2} \cdot n \cdot \tau}\right)}{\left(1-\mathrm{e}^{-\lambda_{2} \cdot \tau}\right)} \cdot \mathrm{e}^{-\lambda_{2} \cdot(t-(n-1) \cdot \tau)}+E_{3} \cdot \frac{\left(1-\mathrm{e}^{-k_{a} \cdot n \cdot \tau}\right)}{\left(1-\mathrm{e}^{-k_{a} \cdot \tau}\right)} \cdot \mathrm{e}^{-k_{a} \cdot(t-(n-1) \cdot \tau)}\right\} \mathrm{d} t
\end{aligned}
$$

